\title{
A Trust Region and Affine Scaling Interior Point Method for Nonconvex Minimization with Linear Inequality Constraints
}

\author{
Thomas F. Coleman And YuYing Li ${ }^{1}$
}

\begin{abstract}
A trust region and affine scaling interior point method (TRAM) is proposed for a general nonlinear minimization with linear inequality constraints [8]. In the proposed approach, a Newton step is derived from the complementarity conditions. Based on this Newton step, a trust region subproblem is formed, and the original objective function is monotonically decreased. Explicit sufficient decrease conditions are proposed for satisfying complementarity, dual feasibility and second order optimality.

The objective of this paper is to establish global and local convergence properties of the proposed trust region and affine scaling interior point method. It is shown that the proposed decrease conditions are sufficient for achieving complementarity, dual feasibility and second order optimality respectively. It is also established that a trust region solution is asymptotically in the interior of the proposed trust region subproblem and a damped trust region step can achieve quadratic convergence.
\end{abstract}

Key words. trust region, interior point method, Dikin-affine scaling, Newton step

1. Introduction. When solving a nonlinear programming problem, the number of function and derivative evaluations is often regarded as the main cost indicator. This cost often greatly surpasses that of linear algebra work. An efficient algorithm for a nonlinear programming problem should require as few evaluations as possible. For convex programming problems, interior point methods have proven to be an efficient approach; see [17] for a comprehensive bibliography on these methods. Using these methods, a small number of iterations is typically required to solve large problems $[1,4,26]$. The quest for similar successful interior point algorithms for nonconvex programming problems has become increasingly important $[13,7,9,10,18,15,3]$.

Negative curvature in a nonconvex programming problem implies that there can be many local minimizers: a computational method is typically able to compute one of them. Assume that an initial feasible point $x_{0}$ is available. An algorithm for which the original objective function is monotonically decreased is desirable in a general nonlinear minimization context. The majority of interior point methods, e.g., a path following (see, e.g.,[16]) or a potential function reduction method (see, e.g., [24]), do not have this monotonicity property. Despite lack of polynomial convergence properties, an affine scaling method, e.g., [12, 1, 4, 26], is the only interior point method which approaches a solution by monotonically decreasing the original objective function.

For various structured problems, affine scaling Newton methods have been proposed $[6,5,19,7,20,18,2,10]$. Using these methods, a sequence of "interior" points $\left\{x_{k}\right\}$, with the objective function values monotonically decreasing, are generated to converge quadratically to a solution. To extend this approach to a general nonlinear programming problem, we consider the problem of minimizing a nonlinear (nonconvex) function subject to linear inequality constraints,

$$
\min _{x \in \Re^{n}} f(x)
$$

\footnotetext{
${ }^{1}$ Research partially supported by the Applied Mathematical Sciences Research Program (KC04-02) of the Office of Energy Research of the U.S. Department of Energy under grant DE-FG0286ER25013.A000, and in part by NSF through grant DMS-9505155, ONR through grant N00014-961-0050, and by the Cornell Theory Center which receives major funding from the National Science Foundation and IBM Corporation, with additional support from New York State and members of its Corporate Research Institute.
} 
subject to $A x \geq b$,

where $A^{T}=\left[a_{1}, \cdots, a_{m}\right] \in \Re^{n \times m}$. In this paper, $\mathcal{F} \stackrel{\text { def }}{=}\{x: A x \geq b\}$ denotes the feasible region. Moreover, it is assumed that the interior of the feasible region $\operatorname{int}(\mathcal{F}) \stackrel{\text { def }}{=}\{x: A x>b\}$ is not empty, $f(x)$ is at least continuously differentiable in $\mathcal{F}$ and twice continuously differentiable if second order convergence is considered. Moreover, a strictly feasible initial point $x_{0} \in \operatorname{int}(\mathcal{F})$ is assumed to be given.

In [8], a trust region and affine scaling interior point method (TRAM) is proposed for (1.1). Given the current interior point $x_{k}$, an improved strictly feasible iterate $x_{k+1} \in \operatorname{int}(\mathcal{F})$ is generated by solving a trust region subproblem with a quadratic objective function and a trust region bound using a 2-norm measurement. Asymptotically, solutions of the trust region subproblems are approximate affine scaling Newton steps for the complementarity conditions.

The main objective of this article is to analyze global and local convergence properties of TRAM. In $\S 2$, the proposed TRAM and its explicit decrease conditions are described. The global convergence properties are established in $\S 3$ and local convergence properties in $\S 4$. Conclusion and future work are discussed in $\S 5$. In [8], an interested reader can find a more detailed motivation for TRAM and its preliminary computational results.

2. The Trust Region and Affine Scaling Interior Point Algorithm. The idea behind a trust region method for unconstrained minimization is intuitive and simple. In a neighborhood of the current point $x_{k}$, a quadratic objective is minimized over a "region of trust" to yield a sufficient decrease of the original objective function. The size of the trust region neighborhood is updated according to agreement of the objective function and its approximation.

Constraints make it difficult to formulate a similar subproblem for which a global solution can be computed by existing software. The algorithm TRAM proposed in [8] uses a trust region mechanism with a 2 -norm trust region bound. In this approach, constraints in the original minimization do not appear in the subproblem explicitly; difficulties of constraints are overcome using affine scaling to formulate an appropriate quadratic function and trust region. We briefly describe TRAM next and refer a reader to [8] for a more detailed discussion.

Let $x_{k}$ be the current strictly feasible iterate and $\lambda_{k}$ be an approximation to the Lagrangian multipliers. Let

$$
C_{k} \stackrel{\text { def }}{=} \operatorname{diag}\left(\left|\lambda_{k}\right|\right), \quad D(x) \stackrel{\text { def }}{=} \operatorname{diag}(A x-b), \quad \text { and } \quad D_{k} \stackrel{\text { def }}{=} D\left(x_{k}\right) .
$$

Assume $\psi_{k}(d)$ denotes the quadratic approximation to $f(x)$ at $x_{k}$, i.e.,

$$
\psi_{k}(d) \stackrel{\text { def }}{=} \nabla f_{k}^{T} d+\frac{1}{2} d^{T} B_{k} d
$$

where $B_{k}$ is symmetric and approximates the Hessian $\nabla^{2} f_{k}$ of $f(x)$ at $x_{k}$.

In [8], a trust region subproblem is derived from the Newton step for the complementarity conditions of (1.1). The complementarity conditions of (1.1), e.g., [14], can be expressed as an $(m+n)$-by- $(m+n)$ system of nonlinear equations

$$
\operatorname{diag}(A x-b) \lambda=0 \quad \text { and } \quad A^{T} \lambda-\nabla f=0 .
$$


Consider the solution $\left(p_{k}^{N} ; \lambda_{k+1}^{p}-\lambda_{k}\right)$ to the following system,

$$
\left[\begin{array}{ll}
\nabla^{2} f_{k} & -A^{T} \\
C_{k} A & D_{k}
\end{array}\right]\left[\begin{array}{l}
p_{k}^{N} \\
\lambda_{k+1}^{p}-\lambda_{k}
\end{array}\right]=-\left[\begin{array}{l}
\nabla f_{k}-A^{T} \lambda_{k} \\
D_{k} \lambda_{k}
\end{array}\right],
$$

It is easy to verify that $\left(p_{k}^{N} ; \lambda_{k+1}^{p}-\lambda_{k}\right)$ is an approximate Newton step for the complementarity condition (2.3) in a neighborhood of a local minimizer of (1.1).

Let $\hat{p}_{k} \stackrel{\text { def }}{=} D_{k}^{-\frac{1}{2}} A p_{k}$. When $\left\|\left(p_{k}^{N} ; \hat{p}_{k}^{N}\right)\right\|_{2}<\Delta_{k},\left(p_{k}^{N} ; \hat{p}_{k}^{N}\right)$ is the Newton step of the following trust region subproblem

$$
\begin{array}{ll} 
& \min _{d \in \Re^{n}, \hat{d} \in \Re^{m}} \nabla f_{k}^{T} d+\frac{1}{2} d^{T} \nabla^{2} f_{k} d+\frac{1}{2} \hat{d}^{T} C_{k} \hat{d} \\
\text { subject to } \quad & A d-D_{k}^{\frac{1}{2}} \hat{d}=0 \\
& \|(d ; \hat{d})\|_{2} \leq \Delta_{k} .
\end{array}
$$

Let $\hat{H}_{k}$ denote the Hessian of (2.5), i.e.,

$$
\hat{H}_{k} \stackrel{\text { def }}{=}\left[\begin{array}{cc}
\nabla^{2} f_{k} & 0 \\
0 & C_{k}
\end{array}\right] .
$$

Let the columns of $Z_{k}$ denote an orthonormal basis for the null space of $\left[A,-D_{k}^{\frac{1}{2}}\right]$. Then the projected Hessian of the subproblem (2.5) is $Z_{k}^{T} \hat{H}_{k} Z_{K}$. The optimality conditions of (2.5) imply that there exists a parameter $\nu_{k} \geq 0$ such that

$$
\left(\hat{H}_{k}+\nu_{k} I\right)\left(p_{k} ; \hat{p}_{k}\right)=-\left[\begin{array}{c}
\nabla f_{k} \\
0
\end{array}\right]+\left[\begin{array}{c}
A^{T} \\
-D_{k}^{\frac{1}{2}}
\end{array}\right] \lambda_{k+1}^{p},
$$

with $\nu_{k}\left(\Delta_{k}-\left\|\left(p_{k} ; \hat{p}_{k}\right)\right\|_{2}\right)=0$. Then $\left(p_{k} ; \hat{p}_{k}\right)=Z_{k} Z_{k}^{T}\left(p_{k} ; \hat{p}_{k}\right)$ and the projected Hessian $Z_{k}^{T}\left(\hat{H}_{k}+\nu_{k} I\right) Z_{k}$ is positive semi-definite, i.e.,

$$
Z_{k}^{T}\left(\hat{H}_{k}+\nu_{k} I\right) Z_{k}=R_{k}^{T} R_{k},
$$

where $R_{k}$ is an upper triangular matrix.

Stepsize choice is important for computational and theoretical convergence behaviors of an affine scaling method for linear programming [21, 25]. One would like to take as large a step as possible but this may bring the iterates to the boundary prematurely. Nonlinearity of the problem (1.1) can both alleviate and exacerbate this problem. On the one hand, the iterates may approach a boundary more slowly due to nonlinearity of $f(x)$. On the other hand, once close to a boundary, nonlinearity of $f(x)$ may make it harder to move away. Two strategies are proposed in [8] to overcome this difficulty. Firstly, a simple reflection technique can be used which facilitates departure from a boundary. We refer an interested reader to [8] for details. Secondly, when close to a non-optimal point satisfying complementarity, a perturbed diagonal scaling $\tilde{D}_{k} \stackrel{\text { def }}{=} \tilde{D}\left(x_{k}\right)$ is used instead, for example,

$$
(\tilde{D}(x))_{i i}= \begin{cases}(D(x))_{i i} & \text { if } i \neq j_{0} \\ 1 & \text { otherwise }\end{cases}
$$


and the iterates are to move away from a constraint $j_{0}$ which can be identified as

$$
(\lambda(x))_{j_{0}}=\min \left\{(\lambda(x))_{i}:\left|a_{i}^{T} x-b_{i}\right|<\sigma \text { and }(\lambda(x))_{i}<0\right\}
$$

where $0<\sigma<1$ is a small constant. When there is no $i$ with $\left|a_{i}^{T} x-b_{i}\right|<\sigma$ and $(\lambda(x))_{i}<0$, it is assumed that $j_{0}=0$ and $D(x)=\tilde{D}(x)$. Geometrically, $\tilde{D}_{k}$ changes the shape of the trust region so that it is elongated along the normal $a_{j_{0}}$ of the $j_{0}$ th constraint. This forces a step away from the nearly binding constraint $j_{0}$.

In general, the trust region subproblem proposed in [8] is,

$$
\begin{aligned}
\min _{d \in \Re^{n}} \psi_{k}(d)+\frac{1}{2} d^{T} A^{T} S_{k}^{-1} C_{k} A d \\
\text { subject to }\left\|\left(d ; S_{k}^{-\frac{1}{2}} d\right)\right\|_{2} \leq \Delta_{k},
\end{aligned}
$$

where $S_{k}$ is an affine scaling matrix which typically equals $D_{k}$. The diagonal scaling $D_{k}^{-\frac{1}{2}}$ in the 2-norm trust region bound constraint serves a similar purpose as Dikin affine scaling [12] for a linear programming problem. The classical Dikin affine scaling uses $D_{k}^{-1}$ rather than $D_{k}^{-\frac{1}{2}}$. The scaling matrix $S_{k}$ in the trust region subproblem (2.10) is typically $D_{k}$ while $\tilde{D}_{k}$ is occasionally used when an iterate is close to a non-optimal point satisfying complementarity.

An approximate trust region solution $p_{k}$ is damped for strict feasibility. Assume $0<\theta_{0}<1$. Let $\theta_{k} \in\left[\theta_{0}, 1\right)$ be a damping parameter. For example, let $p_{k}$ be a solution to the trust region subproblem (2.10). A damped step $p_{k}^{*}$ can be defined as:

$$
p_{k}^{*} \stackrel{\text { def }}{=} \alpha_{k} p_{k} \quad \text { and } \quad \alpha_{k}=\theta_{k} \min \left\{1, \beta_{k}\right\},
$$

where $\beta_{k}$ is the stepsize to the boundary of $\mathcal{F}$ along $p_{k}$. The new iterate $x_{k+1}=x_{k}+p_{k}^{*}$ is computed. The trust region size $\Delta_{k}$ is subsequently adjusted based on the agreement between the original objective function and its approximation. This process is then repeated. TRAM is described in FIG. 1.

The quadratic objective in the subproblem (2.10) is a quadratic approximation to the original objective function augmented by a quadratic term adding positive curvature in the space spanned by the constraint normals. This is similar to an augmented Lagrangian function for a constrained minimization. The presence of the affine scaling in both the objective function and the trust region bound constraint ensures that a damped step of the trust region solution is able to yield a sufficient decrease.

Sufficient decreases conditions are useful in determination of approximate trust region solutions. In unconstrained minimization, sufficient decrease is measured against reductions of the quadratic objective along the gradient direction (a first order approximation to the trust region subproblem) and the solution to a trust region subproblem. For the constrained nonlinear minimization problem (1.1), similar explicit decrease conditions are proposed in [8] based on the trust region subproblem (2.10).

A sufficient decease condition for complementarity (2.3) is derived from the projected gradient direction $g_{k}$ of the trust region subproblem (2.10) with $S_{k}=D_{k}$, i.e.,

$$
g_{k}=-\left(\nabla f_{k}-A^{T} \lambda_{k}\right),
$$


TRAM Let $0<\mu<\eta<1$ and $x_{0} \in \operatorname{int}(\mathcal{F})$.

Step 1 Evaluate $f_{k}, \nabla f_{k}$ and $B_{k} \approx \nabla^{2} f_{k}$; compute an approximate Lagrangian multiplier $\lambda_{k}$ and let $C_{k}=\operatorname{diag}\left(\left|\lambda_{k}\right|\right)$.

Step 2 Compute a step $s_{k}, x_{k}+s_{k} \in \operatorname{int}(\mathcal{F})$, based on the trust region subproblem

$$
\begin{aligned}
\min _{d \in \Re^{n}} \psi_{k}(d)+\frac{1}{2} d^{T} A^{T} S_{k}^{-1} C_{k} A d \\
\text { subject to }\left\|\left[d ; S_{k}^{-\frac{1}{2}} d\right]\right\|_{2} \leq \Delta_{k} .
\end{aligned}
$$

Step 3 Compute

$$
\rho_{k}=\frac{f\left(x_{k}+s_{k}\right)-f\left(x_{k}\right)}{\psi\left(s_{k}\right)} .
$$

Step 4 If $\rho_{k}>\mu$ then set $x_{k+1}=x_{k}+s_{k}$. Otherwise set $x_{k+1}=x_{k}$. Update $\Delta_{k}$ as specified.

\section{Updating Trust Region Size $\Delta_{k}$}

Let $0<\gamma_{1}<1<\gamma_{2}$ be given.

1. If $\rho_{k} \leq \mu$ then set $\Delta_{k+1} \in\left(0, \gamma_{1} \Delta_{k}\right]$.

2. If $\rho_{k} \in(\mu, \eta)$ then set $\Delta_{k+1} \in\left[\gamma_{1} \Delta_{k}, \Delta_{k}\right]$.

3. If $\rho_{k} \geq \eta$ then set $\Delta_{k+1} \in\left[\Delta_{k}, \gamma_{2} \Delta_{k}\right]$.

FIG. 1. A Trust Region and Affine Scaling Interior Point Method

where $\lambda_{k}$ is the least squares approximation to the Lagrangian multipliers, i.e.,

$$
\left[\begin{array}{c}
A^{T} \\
-D_{k}^{\frac{1}{2}}
\end{array}\right] \lambda \stackrel{\mathrm{LS}}{=}\left[\begin{array}{c}
\nabla f_{k} \\
0
\end{array}\right]
$$

Let $P_{k}$ denote the orthogonal projection to the null space of $\left[A,-D_{k}^{\frac{1}{2}}\right]$. Then

$$
\nabla f_{k}^{T} g_{k}=-\left\|P_{k}\left[\begin{array}{c}
\nabla f_{k} \\
0
\end{array}\right]\right\|_{2}^{2}=-\left(\left\|\nabla f_{k}-A \lambda_{k}\right\|_{2}^{2}+\left\|D_{k}^{\frac{1}{2}} \lambda_{k}\right\|_{2}^{2}\right) .
$$

Equation (2.13) suggests that a "good" decrease along the projected gradient $g_{k}$ leads to complementarity: $\lim _{k \rightarrow \infty}\left\|\nabla f_{k}-A \lambda_{k}\right\|_{2}=0$ and $\lim _{k \rightarrow \infty}\left\|D_{k}^{\frac{1}{2}} \lambda_{k}\right\|_{2}=0$.

Define $g_{k}^{*} \stackrel{\text { def }}{=} \alpha_{k} g_{k}$, where $\alpha_{k}=\theta_{k} \alpha_{k}^{*}$, as a damped minimizer of the augmented quadratic objective along $g_{k}$ within the feasible trust region, i.e., $\alpha_{k}^{*}$ solves

$$
\min \psi_{k}\left(\alpha g_{k}\right)+\frac{\alpha^{2}}{2} g_{k}^{T} A^{T} D_{k}^{-1} C_{k} A g_{k}
$$




$$
\begin{array}{cc}
\text { subject to } & \left\|\alpha\left(g_{k} ; D_{k}^{-\frac{1}{2}} A g_{k}\right)\right\|_{2} \leq \Delta_{k} \\
& x_{k}+\alpha g_{k} \in \mathcal{F} .
\end{array}
$$

Due to the affine scaling matrix $D_{k}^{-\frac{1}{2}}$, a sufficient decrease of $\psi_{k}(d)$ measured against the decrease from the damped minimizer $g_{k}^{*}$ should lead to satisfaction of complementarity (2.3). This is expressed as condition (AS.1) in FIG. 2.

Assume that strict complementarity holds at $(x ; \lambda)$ : i.e., $D(x) \lambda=0$ and $\nabla f(x)-$ $A^{T} \lambda=0$ and there is no $i$ such that $\lambda_{i}=a_{i}^{T} x-b_{i}=0$. Then dual feasibility $\lambda \geq 0$ is satisfied if and only if the system

$$
\tilde{D} \tilde{\lambda}=0 \quad \text { and } \quad \nabla f-A^{T} \tilde{\lambda}=0,
$$

is satisfied. To achieve dual feasibility the projected gradient $\tilde{g}_{k} \stackrel{\text { def }}{=} \tilde{g}\left(x_{k}\right)$ is similarly defined,

$$
\tilde{g}(x) \stackrel{\text { def }}{=}-\left(\nabla f(x)-A^{T} \tilde{\lambda}(x)\right), \quad\left[\begin{array}{c}
A^{T} \\
-\tilde{D}(x)^{\frac{1}{2}}
\end{array}\right] \tilde{\lambda}(x) \stackrel{\operatorname{LS}}{=}\left[\begin{array}{c}
\nabla f(x) \\
0
\end{array}\right] .
$$

Under the strict complementarity assumption, dual feasibility is satisfied if and only $\tilde{g}(x)=0$. Let $\tilde{P}_{k}$ denote the orthogonal projection to the null space of $\left[A,-\tilde{D}_{k}^{\frac{1}{2}}\right]$. Then

$$
\nabla f_{k}^{T} \tilde{g}_{k}=-\left\|\tilde{P}_{k}\left[\begin{array}{c}
\nabla f_{k} \\
0
\end{array}\right]\right\|_{2}^{2}=-\left(\left\|\nabla f_{k}-A \tilde{\lambda}_{k}\right\|_{2}^{2}+\left\|\tilde{D}_{k}^{\frac{1}{2}} \tilde{\lambda}_{k}\right\|_{2}^{2}\right)
$$

Equation (2.17) suggests that a "good" decrease along the projected gradient $\tilde{g}_{k}$ can lead to dual feasibility: $\lim _{k \rightarrow \infty}\left\|\nabla f_{k}-A \tilde{\lambda}_{k}\right\|_{2}=0$ and $\lim _{k \rightarrow \infty}\left\|\tilde{D}_{k}^{\frac{1}{2}} \tilde{\lambda}_{k}\right\|_{2}=0$.

The damped minimizer $\tilde{g}_{k}^{*} \stackrel{\text { def }}{=} \alpha_{k} \tilde{g}_{k}, \alpha_{k}=\theta_{k} \alpha_{k}^{*}$, is along the projected gradient $\tilde{g}_{k}$ where $\alpha_{k}^{*}$ solves

$$
\begin{array}{ll} 
& \min \psi_{k}\left(\alpha \tilde{g}_{k}\right)+\frac{\alpha^{2}}{2} \tilde{g}_{k}^{T} A^{T} \tilde{D}_{k}^{-1} C_{k} A \tilde{g}_{k} \\
\text { subject to } \quad & \left\|\alpha\left(\tilde{g}_{k} ; \tilde{D}_{k}^{-\frac{1}{2}} A \tilde{g}_{k}\right)\right\|_{2} \leq \Delta_{k} \\
& x_{k}+\alpha \tilde{g}_{k} \in \mathcal{F} .
\end{array}
$$

This leads to condition (AS.2) in FIG. 2 to ensure dual feasibility.

For second order convergence, sufficient decrease can be measured against the decrease generated by the trust region solution. Condition (AS.3) in FIG. 2 is proposed as sufficient decrease for second order optimality and fast local convergence.

Conditions (AS.1), (AS.2) and (AS.3) in FIG. 2 are proposed as sufficient decrease criteria for complementarity, dual feasibility and second order optimality respectively. Using these conditions, desired convergence properties can be achieved. We refer an interested reader to [8] for an example implementation.

3. Global Convergence. We now establish that conditions (AS.1), (AS.2) and (AS.3) in FIG. 2 are sufficient for TRAM to achieve complementarity, dual feasibility and second order optimality respectively. Equation (2.13) suggests that complementarity can be satisfied if decrease is comparable to that of the minimizer along $g_{k}$. 
Sufficient Decrease Conditions. Let $0<\beta_{c s}, \beta_{d f}, \beta_{q}<1$ and $\beta_{s}>0$. Let $g_{k}, \tilde{g}_{k}$ be defined by (2.12) and (2.16). Let $p_{k}$ be a solution to (2.10) with $S_{k}=D_{k}$ and $B_{k}=\nabla^{2} f_{k}$. Define $g_{k}^{*}, \tilde{g}_{k}^{*}$ and $p_{k}^{*}$ as in (2.14), (2.18) and (2.11) respectively. Let $\left\|s_{k}\right\|_{2} \leq \beta_{s} \Delta_{k}$. The sufficient decrease conditions are:

(AS.1)

$$
\psi_{k}\left(s_{k}\right)<\beta_{c s}\left(\psi_{k}\left(g_{k}^{*}\right)+\frac{1}{2} g_{k}^{* T} A^{T} D_{k}^{-1} C_{k} A g_{k}^{*}\right)
$$

(AS.2)

$$
\psi_{k}\left(s_{k}\right)<\beta_{d f}\left(\psi_{k}\left(\tilde{g}_{k}^{*}\right)+\frac{1}{2} \tilde{g}_{k}^{* T} A^{T} \tilde{D}_{k}^{-1} C_{k} A \tilde{g}_{k}^{*}\right)
$$

(AS.3) $\left(\psi_{k}\left(s_{k}\right)+\frac{1}{2} s_{k}^{T} A^{T} D_{k}^{-1} C_{k} A s_{k}\right)<\beta_{q}\left(\psi_{k}\left(p_{k}^{*}\right)+\frac{1}{2} p_{k}^{* T} A^{T} D_{k}^{-1} C_{k} A p_{k}^{*}\right)$, where $B_{k}=\nabla^{2} f_{k}$.

FIG. 2. Sufficient Decrease Conditions for the First and Second Order Optimality

Therefore condition (AS.1) is sufficient if the damped minimizer $g_{k}^{*}$ retains a significant portion of this minimizer. Similar remarks are applicable to $\tilde{g}_{k}^{*}$ for dual feasibility. An important component of the global convergence analysis is to establish that, due to affine scaling, the posterior damping for feasibility does not prohibit sufficient decrease.

To establish convergence properties of TRAM, the following compactness and full row rank assumption is made.

(AS.0) Given an initial point $x_{0} \in \operatorname{int}(\mathcal{F})$, it is assumed that the level set $\mathcal{L}$ is compact, where $\mathcal{L} \stackrel{\text { def }}{=}\left\{x: x \in \mathcal{F}\right.$ and $\left.f(x) \leq f\left(x_{0}\right)\right\}$. Moreover, $[A,-D(x)]$ is assumed to have full row rank for all $x \in \mathcal{L}$.

The matrix $[A,-D(x)]$ has full row rank if and only if the vectors $\left\{a_{i}: a_{i}^{T} x-b_{i}=\right.$ $0\}$ are linearly independent. In addition $[A,-\tilde{D}(x)]$ has full row rank if $[A,-D(x)]$ has full row rank. Under the full rank assumption, $\lambda_{k}$ and $\tilde{\lambda}_{k}$ can be computed via the normal equations of (2.12) and (2.16), i.e.,

$$
A A^{T} \lambda_{k}+D_{k} \lambda_{k}=A \nabla f_{k}, \quad A A^{T} \tilde{\lambda}_{k}+\tilde{D}_{k} \tilde{\lambda}_{k}=A \nabla f_{k} .
$$

Equation (3.1) gives the relations below which are frequently used in the subsequent analysis:

$$
A g_{k}=-D_{k} \lambda_{k}, \quad A \tilde{g}_{k}=-\tilde{D}_{k} \tilde{\lambda}_{k}
$$

Lemma 3.1. Assume that (AS.0) holds and $f(x): \mathcal{F} \rightarrow \Re$ is continuously differentiable. Let $D(x), \tilde{D}(x), g(x), \tilde{g}(x), \lambda(x)$ and $\tilde{\lambda}(x)$ be defined as in (2.1), (2.8), (2.12) and (2.16). Then

(a) $g(x)$ and $\lambda(x)$ are continuous in $\mathcal{L}$;

(b) $\tilde{g}(x)$ and $\tilde{\lambda}(x)$ are bounded in $\mathcal{L}$;

(c) $D(x)^{-\frac{1}{2}} A g(x)$ and $\tilde{D}(x)^{-\frac{1}{2}} A \tilde{g}(x)$ are bounded in $\mathcal{L}$.

Proof. From (AS.0), $[A,-D(x)]$ has full row rank for any $x$ in $\mathcal{L}$. Then $[A,-\tilde{D}(x)]$ has full row rank for any $x$ in $\mathcal{L}$. Therefore, $\left(A^{T} A+D(x)\right)$ and $\left(A^{T} A+\tilde{D}(x)\right)$ are nonsingular for any $x$ in $\mathcal{L}$. Hence $g(x), \lambda(x), \tilde{g}(x)$ and $\tilde{\lambda}(x)$ are defined for all $x$ in $\mathcal{L}$.

From definition (2.12) and (2.16), $g(x)=-\left(\nabla f(x)-A^{T} \lambda(x)\right)$ and $\tilde{g}(x)=-(\nabla f(x)-$ $\left.A^{T} \tilde{\lambda}(x)\right)$, where $\lambda(x)=\left(A^{T} A+D(x)\right)^{-1} \nabla f(x)$ and $\tilde{\lambda}(x)=\left(A^{T} A+\tilde{D}(x)\right)^{-1} \nabla f(x)$. 
Since $\nabla f(x)$ and $D(x)$ are continuous in $\mathcal{F}$ and $A^{T} A+D(x)$ is nonsingular in $\mathcal{L}, g(x)$ and $\lambda(x)$ are continuous in $\mathcal{L}$. From compactness of $\mathcal{L}, g(x)$ and $\lambda(x)$ are bounded in $\mathcal{L}$. Applying continuity of $\nabla f(x)$ and nonsingularity of $A^{T} A+\tilde{D}(x)$ in the compact set $\mathcal{L}, \tilde{g}(x)$ and $\tilde{\lambda}(x)$ are bounded in $\mathcal{L}$. Using $(3.2), D(x)^{-\frac{1}{2}} A g(x)$ and $\tilde{D}(x)^{-\frac{1}{2}} A \tilde{g}(x)$ are bounded in $\mathcal{L}$. The proof is completed. $\square$

Lemma 3.2 below further describes the relationship between complementarity and the projected gradient $g_{k}$, and dual feasibility and $\tilde{g}_{k}$.

Lemma 3.2. Assume that (AS.O) holds, $\left\{B_{k}\right\}$ is bounded, and $f(x): \mathcal{F} \rightarrow \Re$ is continuously differentiable. Moreover assume that $\nabla f_{*}^{T} g_{*}=0$ at $x_{*} \in \mathcal{L}$. Then for any $\left\{x_{k}\right\}$ in $\mathcal{L}$ which converges to $x_{*},\left\{\nabla f_{k}^{T} g_{k}\right\}$ converges to zero. In addition, if there exists a sequence $\left\{x_{k}\right\}$ in $\mathcal{L}$ converging to $x_{*}$ with $\lim _{k \rightarrow \infty} \nabla f_{k}^{T} \tilde{g}_{k}=0$ and strict complementarity holds at $x_{*}$, then $\left(\lambda_{*}\right)_{i}>0$ for any $a_{i}^{T} x_{*}-b_{i}=0$.

Proof. From Lemma 3.1, $g(x)$ is continuous in $\mathcal{L}$. This implies that $\left\{\nabla f_{k}^{T} g_{k}\right\}$ converges to zero for any $\left\{x_{k}\right\}$ in $\mathcal{L}$ which converges to $x_{*}$.

Assume now that there exists a sequence $\left\{x_{k}\right\}$ in $\mathcal{L}$ converging to $x_{*}$ with $\left\{\nabla f_{k}^{T} \tilde{g}_{k}\right\}$ converging to zero and strict complementarity holds at $x_{*}$. If there exists $\left(\lambda_{*}\right)_{i}<0$ for some $a_{i}^{T} x_{*}-b_{i}=0$, then there exists $j_{0} \neq 0$ with $\left(\tilde{D}_{*}\right)_{j_{0} j_{0}}=1$ and $a_{j_{0}}^{T} x_{*}-b_{j_{0}}=0$. Moreover, from $\lim _{k \rightarrow \infty} \nabla f_{k}^{T} \tilde{g}_{k}=0$, there exists $\tilde{\lambda}_{*}$ such that $\nabla f_{*}=A^{T} \tilde{\lambda}_{*}$ and $\tilde{D}_{*} \tilde{\lambda}_{*}=$ 0 . If $\tilde{\lambda}_{*}=\lambda_{*}$, strict complementarity is violated since $\left(\tilde{\lambda}_{*}\right)_{j_{0}}=0$. If $\tilde{\lambda}_{*} \neq \lambda_{*}$, the full row rank assumption is violated since $\left[A,-D_{*}\right]^{T} \tilde{\lambda}_{*}=\left(\nabla f_{*} ; 0\right)$ and $\left[A,-D_{*}\right]^{T} \lambda_{*}=$ $\left(\nabla f_{*} ; 0\right)$. Hence $\left(\lambda_{*}\right)_{i}>0$ for any $a_{i}^{T} x_{*}-b_{i}=0$. The proof is completed.

Lemma 3.3 relates decrease required by (AS.1) and (AS.2) with complementarity and dual feasibility. Its proof is similar to Lemma (4.8) in [22].

Lemma 3.3. Assume that $\left\{x_{k}\right\}$ is generated from TRAM, (AS.O) is satisfied, and $f(x): \mathcal{F} \rightarrow \Re$ is continuously differentiable and $\left\{B_{k}\right\}$ is bounded. If (AS.1) is satisfied at the $k$ th iteration, then there exist constants $\chi_{\beta}, \chi_{\nu}, \chi_{g}>0$ such that

$$
-\psi_{k}\left(s_{k}\right) \geq \frac{\beta_{c s} \theta_{0}^{2}}{2 \chi_{g}}\left(-\nabla f_{k}^{T} g_{k}\right) \min \left\{\Delta_{k}, \frac{-\left(\nabla f_{k}^{T} g_{k}\right)}{\chi_{\nu} \chi_{g}}, \chi_{\beta} \sqrt{\left\|g_{k}\right\|_{2}^{2}+\left\|D_{k}^{\frac{1}{2}} \lambda_{k}\right\|_{2}^{2}}\right\} .
$$

If (AS.2) is satisfied and $\left(\tilde{\lambda}_{k}\right)_{j_{0}}<0$, then there exist $\chi_{\tilde{\beta}}, \chi_{\nu}, \chi_{\tilde{g}}>0$ such that

$$
-\psi_{k}\left(s_{k}\right) \geq \frac{\beta_{d f} \theta_{0}^{2}}{2 \chi_{\tilde{g}}}\left(-\nabla f_{k}^{T} \tilde{g}_{k}\right) \min \left\{\Delta_{k}, \frac{-\left(\nabla f_{k}^{T} \tilde{g}_{k}\right)}{\chi_{\nu} \chi_{\tilde{g}}}, \chi_{\tilde{\beta}} \sqrt{\left\|\tilde{g}_{k}\right\|_{2}^{2}+\left\|\tilde{D}_{k}^{\frac{1}{2}} \tilde{\lambda}_{k}\right\|_{2}^{2}}\right\} .
$$

Here the index $j_{0}$ is as defined in (2.9).

Proof. Define $\phi(\tau): \Re \rightarrow \Re$ by

$$
\phi(\tau)=\psi_{k}\left(\tau d_{k}\right)+\frac{\tau^{2}}{2} d_{k}^{T} A^{T} D_{k}^{-1} C_{k} A d_{k} \quad \text { if } \quad d_{k}=\frac{g_{k}}{\sqrt{\left\|g_{k}\right\|_{2}^{2}+\left\|D_{k}^{-\frac{1}{2}} A g_{k}\right\|_{2}^{2}}},
$$

and

$$
\phi(\tau)=\psi_{k}\left(\tau d_{k}\right)+\frac{\tau^{2}}{2} d_{k}^{T} A^{T} \tilde{D}_{k}^{-1} C_{k} A d_{k} \quad \text { if } \quad d_{k}=\frac{\tilde{g}_{k}}{\sqrt{\left\|\tilde{g}_{k}\right\|_{2}^{2}+\left\|\tilde{D}_{k}^{-\frac{1}{2}} A \tilde{g}_{k}\right\|_{2}^{2}}} .
$$


Hence $\phi(\tau)=\tau\left(\nabla f_{k}^{T} d_{k}\right)+\frac{1}{2} \tau^{2} \nu_{k}$, where

$$
\nu_{k}=d_{k}^{T} B_{k} d_{k}+d_{k}^{T} A^{T} D_{k}^{-1} C_{k} A d_{k}, \quad \text { if } \quad d_{k}=\frac{g_{k}}{\sqrt{\left\|g_{k}\right\|_{2}^{2}+\left\|D_{k}^{-\frac{1}{2}} A g_{k}\right\|_{2}^{2}}},
$$

and

$$
\nu_{k}=d_{k}^{T} B_{k} d_{k}+d_{k}^{T} A^{T} \tilde{D}_{k}^{-1} C_{k} A d_{k}, \quad \text { if } \quad d_{k}=\frac{\tilde{g}_{k}}{\sqrt{\left\|\tilde{g}_{k}\right\|_{2}^{2}+\left\|\tilde{D}_{k}^{-\frac{1}{2}} A \tilde{g}_{k}\right\|_{2}^{2}}} .
$$

Since $\left\{B_{k}\right\},\left\{\lambda_{k}\right\}$ and $\left\{\tilde{\lambda}_{k}\right\}$ are bounded, there exists $\chi_{\nu}>0$ such that $\left|\nu_{k}\right| \leq \chi_{\nu}$. Let $\tau_{k}^{*}$ be the minimizer of $\phi(\tau)$ on $\left[0, \min \left\{\Delta_{k}, \beta_{k}\right\}\right]$ where $\beta_{k}$ is the stepsize along $d_{k}$ to the boundary of $\mathcal{F}$, i.e.,

$$
\beta_{k}=\min \left\{-\frac{a_{i}^{T} x_{k}-b_{i}}{a_{i}^{T} d_{k}}:-\frac{a_{i}^{T} x_{k}-b_{i}}{a_{i}^{T} d_{k}}>0\right\} .
$$

The stepsize $\beta_{k} \stackrel{\text { def }}{=}+\infty$ if $-\frac{a_{i}^{T} x_{k}-b_{i}}{a_{i}^{T} d_{k}} \leq 0$ for all $i$. If $\tau_{k}^{*} \in\left[0, \min \left\{\Delta_{k}, \beta_{k}\right\}\right)$, then $0 \leq \nu_{k} \leq \chi_{\nu}$ and $\tau_{k}^{*}=-\left(\nabla f_{k}^{T} d_{k}\right) / \nu_{k}$. From $0<\theta_{0} \leq \theta_{k}<1$,

$$
\phi\left(\theta_{k} \tau_{k}^{*}\right)=-\theta_{k} \frac{\left(\nabla f_{k}^{T} d_{k}\right)^{2}}{\nu_{k}}+\frac{1}{2} \theta_{k}^{2} \frac{\left(\nabla f_{k}^{T} d_{k}\right)^{2}}{\nu_{k}} \leq-\frac{\theta_{0}^{2}}{2} \frac{\left(\nabla f_{k}^{T} d_{k}\right)^{2}}{\chi_{\nu}} .
$$

Assume $\tau_{k}^{*}=\Delta_{k}$. Since $\nu_{k} \Delta_{k} \leq-\left(\nabla f_{k}^{T} d_{k}\right)$ when $\nu_{k}>0$, and $\phi\left(\theta_{k} \tau_{k}^{*}\right) \leq \theta_{0}^{2} \Delta_{k}\left(\nabla f_{k}^{T} d_{k}\right)$ otherwise,

$$
\phi\left(\theta_{k} \tau_{k}^{*}\right)=\phi\left(\theta_{k} \Delta_{k}\right) \leq \frac{\theta_{0}^{2}}{2} \Delta_{k}\left(\nabla f_{k}^{T} d_{k}\right)
$$

Assume $\tau_{k}^{*}=\beta_{k}$. Since $\nu_{k} \beta_{k} \leq-\left(\nabla f_{k}^{T} d_{k}\right)$ when $\nu_{k}>0$, and $\phi\left(\theta_{k} \tau_{k}^{*}\right) \leq \theta_{0}^{2} \beta_{k}\left(\nabla f_{k}^{T} d_{k}\right)$ otherwise,

$$
\phi\left(\theta_{k} \tau_{k}^{*}\right)=\phi\left(\theta_{k} \beta_{k}\right) \leq \frac{\theta_{0}^{2}}{2} \beta_{k}\left(\nabla f_{k}^{T} d_{k}\right) .
$$

Considering all the three cases,

$$
\phi_{k}\left(\theta_{k} \alpha_{k}^{*} d_{k}\right) \leq \frac{\theta_{0}^{2}}{2}\left(\nabla f_{k}^{T} d_{k}\right) \min \left\{\Delta_{k}, \frac{-\left(\nabla f_{k}^{T} d_{k}\right)}{\chi_{\nu}}, \beta_{k}\right\} .
$$

Next we establish lower bounds for $\beta_{k}$ for the two definitions of $d_{k}$ respectively.

First consider $d_{k}=g_{k} / \sqrt{\left\|g_{k}\right\|_{2}^{2}+\left\|D_{k}^{-\frac{1}{2}} A g_{k}\right\|_{2}^{2}}$. From (3.2), boundedness of $\left\{\lambda_{k}\right\}$ and $\left\{x_{k}\right\}$, there exists $\chi_{\beta}>0$ such that, for some $1 \leq j \leq m$,

$$
\beta_{k} \geq\left|\frac{a_{j}^{T} x_{k}-b_{j}}{a_{j}^{T} d_{k}}\right|=\left|\frac{\sqrt{\left\|g_{k}\right\|_{2}^{2}+\left\|D_{k}^{-\frac{1}{2}} A g_{k}\right\|_{2}^{2}}}{\left(\lambda_{k}\right)_{j}}\right| \geq \chi_{\beta} \sqrt{\left\|g_{k}\right\|_{2}^{2}+\left\|D_{k}^{\frac{1}{2}} \lambda_{k}\right\|_{2}^{2}} .
$$

If $d_{k}=\tilde{g}_{k} / \sqrt{\left\|\tilde{g}_{k}\right\|_{2}^{2}+\left\|\tilde{D}_{k}^{-\frac{1}{2}} A \tilde{g}_{k}\right\|_{2}^{2}}$, from $\left(\tilde{\lambda}_{k}\right)_{j_{0}}<0$, there exists some $j \neq j_{0}$ such that

$$
\beta_{k} \geq\left|\frac{a_{j}^{T} x_{k}-b_{j}}{a_{j}^{T} d_{k}}\right| .
$$


From $(3.2),\left(\tilde{D}_{k}\right)_{i i}=\left(D_{k}\right)_{i i}$ when $i \neq j_{0}$, there exists $\chi_{\tilde{\beta}}>0$ such that

$$
\beta_{k} \geq \chi_{\tilde{\beta}} \sqrt{\left\|\tilde{g}_{k}\right\|_{2}^{2}+\left\|\tilde{D}_{k}^{\frac{1}{2}} \tilde{\lambda}_{k}\right\|_{2}^{2}}
$$

Using Lemma 3.1 and (3.2), there exist $\chi_{g}, \chi_{\tilde{g}}>0$ such that

$$
\sqrt{\left\|g_{k}\right\|_{2}^{2}+\left\|D_{k}^{-\frac{1}{2}} A g_{k}\right\|_{2}^{2}} \leq \chi_{g} \quad \text { and } \quad \sqrt{\left\|\tilde{g}_{k}\right\|_{2}^{2}+\left\|\tilde{D}_{k}^{-\frac{1}{2}} A \tilde{g}_{k}\right\|_{2}^{2}} \leq \chi_{\tilde{g}} \text {. }
$$

Since $\psi_{k}\left(s_{k}\right) \leq \beta_{c s} \phi\left(\theta_{k} \alpha_{k}^{*}\right)$ with $d_{k}=g_{k} / \sqrt{\left\|g_{k}\right\|_{2}^{2}+\left\|D_{k}^{-\frac{1}{2}} A g_{k}\right\|_{2}^{2}}$ if (AS.1) is satisfied and $\psi_{k}\left(s_{k}\right) \leq \beta_{d f} \phi\left(\theta_{k} \alpha_{k}^{*}\right)$ with $d_{k}=\tilde{g}_{k} / \sqrt{\left\|\tilde{g}_{k}\right\|_{2}^{2}+\left\|\tilde{D}_{k}^{-\frac{1}{2}} A \tilde{g}_{k}\right\|_{2}^{2}}$, if (AS.2) is satisfied, the results follow from $(3.6),(3.7),(3.8)$, and (3.9). $\square$

Lemma 3.4. Assume that (AS.O) holds, $f(x): \mathcal{F} \rightarrow \Re$ is continuously differentiable, $\left\{B_{k}\right\}$ is bounded and $\left\{x_{k}\right\}$ is generated by TRAM. Then there cannot exist constants $\chi_{0}, \chi_{1}>0$ such that, for sufficiently large $k$,

$$
-\psi_{k}\left(s_{k}\right) \geq \frac{1}{2} \chi_{0} \min \left\{\Delta_{k}, \chi_{1}\right\}
$$

Proof. The lemma is proved by contradiction. Assume that there exist constants $\chi_{0}, \chi_{1}>0$ such that, for sufficiently large $k$,

$$
-\psi_{k}\left(s_{k}\right) \geq \frac{1}{2} \chi_{0} \min \left\{\Delta_{k}, \chi_{1}\right\} .
$$

We first prove that

$$
\sum_{k=1}^{\infty} \Delta_{k}<+\infty .
$$

If there are a finite number of successful iterations then $\Delta_{k+1} \leq \gamma_{1} \Delta_{k}$ for all $k$ sufficiently large. Hence

$$
\sum_{k=1}^{\infty} \Delta_{k}<+\infty .
$$

If there is an infinite sequence $\left\{k_{i}\right\}$ of successful iterations, using (3.10), for a successful step and sufficiently large $k$,

$$
f\left(x_{k_{i}}\right)-f\left(x_{k_{i}+1}\right) \geq-\mu \psi_{k}\left(s_{k_{i}}\right) \geq \frac{1}{2} \mu \chi_{0} \min \left\{\Delta_{k_{i}}, \chi_{1}\right\} .
$$

Since $f\left(x_{k+1}\right) \leq f\left(x_{k}\right)$ for all $k$ and $\left\{f\left(x_{k}\right)\right\}$ is bounded below,

$$
\sum_{i=1}^{\infty} \Delta_{k_{i}}<+\infty
$$

The trust region updating rules of TRAM imply that

$$
\sum_{k=1}^{\infty} \Delta_{k} \leq\left(1+\frac{\gamma_{2}}{1-\gamma_{1}}\right) \sum_{i=1}^{\infty} \Delta_{k_{i}} .
$$


Again (3.11) holds.

From (3.11) and $\left\|x_{k+1}-x_{k}\right\|_{2}=\left\|s_{k}\right\|_{2} \leq \beta_{s} \Delta_{k},\left\{x_{k}\right\}$ converges and $\left\{s_{k}\right\}$ converges to zero. Since $\left\{B_{k}\right\}$ is bounded, there exists $\chi_{B}>0$ such that $\left\|B_{k}\right\|_{2} \leq \chi_{B}$. Using $f\left(x_{k}+s_{k}\right)-f\left(x_{k}\right)=\nabla f\left(x_{k}+\xi_{k} s_{k}\right)^{T} s_{k}$ with $0 \leq \xi_{k} \leq 1$,

$$
\begin{aligned}
\left|f\left(x_{k}+s_{k}\right)-f\left(x_{k}\right)-\psi_{k}\left(s_{k}\right)\right| & \leq\left|\frac{1}{2} s_{k}^{T} B_{k} s_{k}\right|+\left|\left(\nabla f\left(x_{k}+\xi_{k} s_{k}\right)-\nabla f_{k}\right)^{T} s_{k}\right| \\
& \leq \frac{1}{2} \chi_{B} \beta_{s}^{2} \Delta_{k}^{2}+\beta_{s} \Delta_{k}\left\|\nabla f\left(x_{k}+\xi_{k} s_{k}\right)-\nabla f_{k}\right\|_{2} .
\end{aligned}
$$

Since $\nabla f(x)$ is continuous in the compact level set $\mathcal{L}$ and $\left\{x_{k}\right\}$ converges, there exists a sequence $\left\{\epsilon_{k}\right\}$ converging to zero such that

$$
\left|f\left(x_{k}+s_{k}\right)-f\left(x_{k}\right)-\psi_{k}\left(s_{k}\right)\right| \leq \epsilon_{k} \Delta_{k} .
$$

By assumption, for sufficiently large $k,-\psi_{k}\left(s_{k}\right) \geq \frac{1}{2} \chi_{0} \Delta_{k}$. It is readily obtained that $\left\{\left|\rho_{k}-1\right|\right\}$ converges to zero. Hence $\left\{\Delta_{k}\right\}$ cannot converge to zero, which is a contradiction to (3.11). The proof is completed. $\square$

Lemma 3.5. Assume that (AS.O) holds and $f(x): \mathcal{F} \rightarrow \Re$ is continuously differentiable. Assume further that $\lim _{k \rightarrow \infty} \nabla f_{k}^{T} g_{k}=0$ and $\liminf _{i \rightarrow \infty}\left(-\nabla f_{m_{i}}^{T} \tilde{g}_{m_{i}}\right)>0$ for a subsequence $\left\{m_{i}\right\}$. Then $\left(\tilde{\lambda}_{m_{i}}\right)_{j_{0}}<0$ for sufficiently large $i$, where $j_{0}$ is defined by (2.9).

Proof. Using (3.2),

$$
\begin{aligned}
\nabla f_{k}^{T} \tilde{g}_{k} & =\left(A^{T} \lambda_{k}\right)^{T} \tilde{g}_{k}+\left(\nabla f_{k}-A \lambda_{k}\right)^{T} \tilde{g}_{k} \\
& =-\lambda_{k}^{T} \tilde{D}_{k} \tilde{\lambda}_{k}+\left(\nabla f_{k}-A \lambda_{k}\right)^{T} \tilde{g}_{k} .
\end{aligned}
$$

From $\lim _{k \rightarrow \infty} \nabla f_{k}^{T} g_{k}=0$ and $(2.13), \lim _{k \rightarrow \infty}\left(\nabla f_{k}-A \lambda_{k}\right)=0$ and $\lim _{k \rightarrow \infty}\left(\lambda_{k}\right)_{j}\left(\tilde{D}_{k}\right)_{j j}=$ 0 for all $j \neq j_{0}$. Since $\liminf _{i \rightarrow \infty}\left(-\nabla f_{m_{i}}^{T} \tilde{g}_{m_{i}}\right)>0,\left(\lambda_{m_{i}}\right)_{j_{0}}\left(\tilde{\lambda}_{m_{i}}\right)_{j_{0}}>0$ for sufficiently large $i$. From $\lim _{k \rightarrow \infty} \nabla f_{k}^{T} g_{k}=0$ and $\liminf _{i \rightarrow \infty}\left(-\nabla f_{m_{i}}^{T} \tilde{g}_{m_{i}}\right)>0,\left(\lambda_{m_{i}}\right)_{j_{0}}<0$ for sufficiently large $i$. This implies that $\left(\tilde{\lambda}_{m_{i}}\right)_{j_{0}}<0$ for sufficiently large $i$.

Lemma 3.6. Assume that (AS.0) holds, $f(x): \mathcal{F} \rightarrow \Re$ is continuously differentiable, and $\left\{B_{k}\right\}$ is bounded.

(a) If (AS.1) holds at every iteration then $\liminf _{k \rightarrow \infty} \nabla f_{k}^{T} g_{k}=0$.

(b) If (AS.2) holds for sufficiently large $k$ and $\lim _{k \rightarrow \infty} \nabla f_{k}^{T} g_{k}=0$, then $\liminf _{k \rightarrow \infty} \nabla f_{k}^{T} \tilde{g}_{k}=0$.

Proof. We prove each result by contradiction.

(a) Assume that there is $\epsilon>0$ such that $-\nabla f_{k}^{T} g_{k} \geq \epsilon$ for all sufficiently large $k$. From (2.13), there exists $\bar{\epsilon}>0$ such that, for sufficiently large $k$,

$$
\left\|\nabla f_{k}-A \lambda_{k}\right\|_{2}^{2}+\left\|D_{k}^{\frac{1}{2}} \lambda_{k}\right\|_{2}^{2} \geq \bar{\epsilon} .
$$

Since (AS.1) is satisfied, using Lemma 3.3 there exist $\chi_{0}, \chi_{1}>0$ such that, for sufficiently large $k$,

$$
-\psi_{k}\left(s_{k}\right) \geq \frac{1}{2} \chi_{0} \min \left\{\Delta_{k}, \chi_{1}\right\}
$$

But Lemma 3.4 indicates that this is not possible. Hence

$$
\liminf _{k \rightarrow \infty} \nabla f_{k}^{T} g_{k}=0 \text {. }
$$


(b) Assume now that (AS.2) holds for sufficiently large $k$ and $\lim _{k \rightarrow \infty} \nabla f_{k}^{T} g_{k}=0$ but $-\nabla f_{k}^{T} \tilde{g}_{k} \geq \epsilon$ for sufficiently large $k$. From (2.17), there exists $\bar{\epsilon}>0$ such that, for sufficiently large $k$,

$$
\left\|\nabla f_{k}-A \tilde{\lambda}_{k}\right\|_{2}^{2}+\left\|\tilde{D}_{k}^{\frac{1}{2}} \tilde{\lambda}_{k}\right\|_{2}^{2} \geq \bar{\epsilon}
$$

Lemma 3.5 and 3.3 apply and there exist $\chi_{0}, \chi_{1}>0$ such that, for sufficiently large $k$,

$$
-\psi_{k}\left(s_{k}\right) \geq \frac{1}{2} \chi_{0} \min \left\{\Delta_{k}, \chi_{1}\right\}
$$

Using Lemma 3.4, this is impossible. Hence

$$
\liminf _{k \rightarrow \infty} \nabla f_{k}^{T} \tilde{g}_{k}=0
$$

The proof is completed.

The next theorem establishes that complementarity and dual feasibility can be satisfied at every limit point.

Theorem 3.7. Assume that (AS.O) holds and $f(x): \mathcal{F} \rightarrow \Re$ is continuously differentiable and $\left\{B_{k}\right\}$ is bounded. Let $\left\{x_{k}\right\}$ be generated by TRAM. Assume that (AS.1) holds at every iteration. Then

(a) $\lim _{k \rightarrow \infty} \nabla f_{k}^{T} g_{k}=0$, i.e., the complementarity conditions are satisfied at every limit point of $\left\{x_{k}\right\}$;

(b) If (AS.2) is satisfied for sufficiently large $k$, then $\lim _{\inf _{k \rightarrow \infty}} \nabla f_{k}^{T} \tilde{g}_{k}=0$. If, in addition, there exists a limit point $x_{*}$ at which strict complementarity is satisfied and $\nabla f_{*}^{T} \tilde{g}_{*}=0$, then $\lim _{k \rightarrow \infty} \nabla f_{k}^{T} \tilde{g}_{k}=0$. Hence, the first order necessary conditions are satisfied at every limit point satisfying strict complementarity.

Proof. The proof is again by contradiction. We consider each result in turn.

(a) Let $\epsilon_{1}$ in $(0,1)$ be given and assume that there is a sequence $\left\{m_{i}\right\}$ such that $\left\|\nabla f_{m_{i}}^{T} g_{m_{i}}\right\|_{2} \geq \epsilon_{1}$. Lemma 3.6 guarantees that for any $\epsilon_{2}$ in $\left(0, \epsilon_{1}\right)$ there is a subsequence of $\left\{m_{i}\right\}$ (without loss of generality we assume that it is the full sequence) and a subsequence $\left\{l_{i}\right\}$ such that

$$
\left|\nabla f_{k}^{T} g_{k}\right| \geq \epsilon_{1}, \quad m_{i} \leq k<l_{i}, \quad\left|\nabla f_{l_{i}}^{T} g_{l_{i}}\right|<\epsilon_{2} .
$$

Using (2.13), there exists $\bar{\epsilon}_{1}>0$ such that, for sufficiently large $i$,

$$
\left\|\nabla f_{k}-A \lambda_{k}\right\|_{2}^{2}+\left\|D_{k}^{\frac{1}{2}} \lambda_{k}\right\|_{2}^{2} \geq \bar{\epsilon}_{1}, \quad m_{i} \leq k<l_{i} .
$$

If the $k$-th iteration is successful, using Lemma 3.3,

$$
f\left(x_{k}\right)-f\left(x_{k+1}\right) \geq \frac{\mu \epsilon_{1} \beta_{c s} \theta_{0}^{2}}{2 \chi_{g}} \min \left\{\Delta_{k}, \frac{\epsilon_{1}}{\chi_{\nu} \chi_{g}}, \chi_{\beta} \bar{\epsilon}_{1}\right\}, \quad m_{i} \leq k<l_{i}
$$

for sufficiently large $i$. Since $f(x)$ is bounded below on $\mathcal{L},\left\{f\left(x_{k}\right)\right\}$ converges and $\left\{f\left(x_{k}\right)-f\left(x_{k+1}\right)\right\}$ converges to zero. From $\left\|x_{k+1}-x_{k}\right\|_{2} \leq \beta_{s} \Delta_{k}$, it follows that, for sufficiently large $i$,

$$
f\left(x_{k}\right)-f\left(x_{k+1}\right) \geq \epsilon_{3}\left\|x_{k+1}-x_{k}\right\|_{2}, \quad m_{i} \leq k<l_{i},
$$


where $\epsilon_{3}=\frac{\mu \epsilon_{1} \beta_{c s} \theta_{0}^{2}}{2 \chi_{g} \beta_{s}}$. Consider a subsequence of $\left\{l_{i}\right\}$ such that $\left\{x_{l_{i}}\right\}$ converges to $x_{*}$ and $\liminf \operatorname{in}_{i \rightarrow \infty} \nabla f_{l_{i}}^{T} g_{l_{i}}=0$. Without loss of generality, the subsequence is still denoted by $\left\{l_{i}\right\}$. Using (3.15) and the triangle inequality,

$$
f\left(x_{m_{i}}\right)-f\left(x_{k_{i}}\right) \geq \epsilon_{3}\left\|x_{k_{i}}-x_{m_{i}}\right\|_{2}, \quad m_{i} \leq k_{i} \leq l_{i} .
$$

Since $\left\{f\left(x_{k}\right)\right\}$ converges and $\left\{x_{l_{i}}\right\}$ converges to $x_{*},\left\{x_{m_{i}}\right\}$ converges to $x_{*}$. From $\left\{\nabla f_{l_{i}}^{T} g_{l_{i}}\right\}$ converging to zero, $\nabla f_{*}^{T} g_{*}=0$. Applying Lemma $3.2,\left\{\nabla f_{m_{i}}^{T} g_{m_{i}}\right\}$ converges to zero. Hence $\left|\nabla f_{m_{i}}^{T} g_{m_{i}}\right|<\epsilon_{2}$ for sufficiently large $i$. This contradicts (3.13) since $\epsilon_{2}<\epsilon_{1}$. Hence $\left\{\nabla f_{k}^{T} g_{k}\right\}$ converges to zero and the complementarity conditions are satisfied at every limit point of $\left\{x_{k}\right\}$;

(b) From (a), $\lim _{k \rightarrow \infty} \nabla f_{k}^{T} g_{k}=0$. Assume now that (AS.2) is satisfied for sufficiently large $k$. Using Lemma 3.6, $\liminf _{k \rightarrow \infty} \nabla f_{k}^{T} \tilde{g}_{k}=0$. The proof for $\lim _{k \rightarrow \infty} \nabla f_{k}^{T} \tilde{g}_{k}=0$ is exactly the same as the proof of (a) except that $g_{k}$ is replaced by $\tilde{g}_{k}$, and (3.14) follows from Lemma 3.5 and Lemma 3.3 with $\beta_{c s}$ replaced by $\beta_{d f}$. Similarly consider a subsequence $\left\{x_{l_{i}}\right\}$ which converges to $x_{*}$ satisfying strict complementarity and $\lim \inf _{i \rightarrow \infty} \nabla f_{l_{i}}^{T} g_{l_{i}}=0$. A contradiction can be derived using Lemma 3.2. Hence $\lim _{k \rightarrow \infty} \nabla f_{k}^{T} \tilde{g}_{k}=0$. Thus dual feasibility is satisfied at every limit point satisfying strict complementarity.

The proof is completed.

Before we examine second order convergence, several technical lemmas are required. First, we quote Lemma (4.10) in [23] below.

Lemma 3.8. Let $x_{*}$ be an isolated limit point of a sequence $\left\{x_{k}\right\}$ in $\Re^{n}$. If $\left\{x_{k}\right\}$ does not converge then there is a subsequence $\left\{x_{l_{j}}\right\}$ converging to $x_{*}$, and an $\epsilon>0$ such that $\left\|x_{l_{j}+1}-x_{l_{j}}\right\|_{2} \geq \epsilon$.

Consequences of (AS.3) are subsequently examined.

Lemma 3.9. Assume that $f(x): \mathcal{F} \rightarrow \Re$ is twice continuously differentiable. Let the columns of $Z_{k}$ form an orthonormal basis for the null space of $\left[A,-D_{k}^{\frac{1}{2}}\right]$ and $p_{k}$ be a solution to the trust region subproblem (2.10) with $S_{k}=D_{k}$ and $B_{k}=\nabla^{2} f_{k}$. Then

$$
-\left(\psi_{k}\left(s_{k}\right)+\frac{1}{2} s_{k}^{T} A^{T} D_{k}^{-1} C_{k} A s_{k}\right) \geq \frac{\beta_{q} \theta_{0}^{2} \min \left\{1, \beta_{k}^{2}\right\}}{2}\left(\nu_{k} \Delta_{k}^{2}+\left\|R_{k} Z_{k}^{T}\left(p_{k} ; \hat{p}_{k}\right)\right\|_{2}^{2}\right),
$$

where $\beta_{k}$ is the stepsize along $p_{k}$ to the boundary of $\mathcal{F}, \nu_{k} \geq 0$ and $R_{k}$ are as defined in (2.6) and (2.7).

Proof. Let $\phi(\alpha)=\psi_{k}\left(\alpha p_{k}\right)+\frac{\alpha^{2}}{2} p_{k}^{T} A^{T} D_{k}^{-1} C_{k} A p_{k}$ and $\alpha \in\left[0, \min \left\{1, \beta_{k}\right\}\right]$ where $\beta_{k}$ is the stepsize along $p_{k}$ to the boundary of $\mathcal{F}$. Let $\alpha_{k}^{*}$ be the minimizer of $\phi(\alpha)$ in $\left[0, \min \left\{1, \beta_{k}\right\}\right]$. Then

$$
\begin{array}{rlr}
\phi(\alpha) & =\alpha \nabla f_{k}^{T} p_{k}+\frac{1}{2} \alpha^{2}\left(p_{k} ; \hat{p}_{k}\right)^{T} \hat{H}_{k}\left(p_{k} ; \hat{p}_{k}\right) & \\
& =-\alpha\left(p_{k} ; \hat{p}_{k}\right)^{T}\left(\hat{H}_{k}+\nu_{k} I\right)\left(p_{k} ; \hat{p}_{k}\right)+\frac{1}{2} \alpha^{2}\left(p_{k} ; \hat{p}_{k}\right)^{T} \hat{H}_{k}\left(p_{k} ; \hat{p}_{k}\right) & (\text { from }(2.6)) \\
& =-\alpha\left\|R_{k} Z_{k}^{T}\left(p_{k} ; \hat{p}_{k}\right)\right\|_{2}^{2}+\frac{1}{2} \alpha^{2}\left\|R_{k} Z_{k}^{T}\left(p_{k} ; \hat{p}_{k}\right)\right\|_{2}^{2}-\frac{1}{2} \alpha^{2} \nu_{k} \Delta_{k}^{2}, \quad(\text { from }(2.7)) .
\end{array}
$$


But $\alpha_{k}^{*}=\min \left\{1, \beta_{k}\right\}$. Hence $\alpha_{k}^{* 2} \leq \alpha_{k}^{*}$. From (AS.3), $p_{k}^{*}=\theta_{k} \alpha_{k}^{*} p_{k}$, and $0<\theta_{0} \leq \theta_{k}<$ 1 ,

$$
-\left(\psi_{k}\left(s_{k}\right)+\frac{1}{2} s_{k}^{T} A^{T} D_{k}^{-1} C_{k} A s_{k}\right) \geq \frac{\beta_{q} \theta_{0}^{2} \min \left\{1, \beta_{k}^{2}\right\}}{2}\left(\nu_{k} \Delta_{k}^{2}+\left\|R_{k} Z_{k}^{T}\left(p_{k} ; \hat{p}_{k}\right)\right\|_{2}^{2}\right) .
$$

The proof is completed.

The following lemma provides lower bounds for the stepsize sequence $\left\{\beta_{k}\right\}$. The results in Lemma 3.10 hold for any subsequence generated by TRAM (consequently, it holds for the entire sequence as well).

Lemma 3.10. Assume that (AS.0) holds, $f(x): \mathcal{F} \rightarrow \Re$ is twice continuously differentiable. Let $\left\{x_{k}\right\}$ be any subsequence generated by TRAM and $\beta_{k}$ be the stepsize along $p_{k}$ to the boundary of $\mathcal{F}$. Then there exist $\chi_{0}, \chi_{1}, \chi_{2}>0$ such that, for sufficiently large $k$,

$$
\beta_{k} \geq \frac{\nu_{k}}{\chi_{0}\left(\chi_{1}+\left(\chi_{2}+\nu_{k}\right) \Delta_{k}\right)}
$$

where $\nu_{k} \geq 0$ is defined in (2.6). Assume further that $\left\{x_{k}\right\}$ converges to $x_{*}$ at which strict complementarity and dual feasibility are satisfied, and $\left\{\left(p_{k} ; \hat{p}_{k}\right)\right\}$ converges to zero. Then $\lim \inf _{k \rightarrow \infty} \beta_{k}>0$.

Proof. By definition,

$$
\beta_{k} \geq \min \left\{-\frac{a_{i}^{T} x_{k}-b_{i}}{a_{i}^{T} p_{k}}:-\frac{a_{i}^{T} x_{k}-b_{i}}{a_{i}^{T} p_{k}}>0\right\}
$$

From $\hat{p}_{k}=\left(A x_{k}-b\right)^{-\frac{1}{2}} A p_{k}$ and $(2.6)$,

$$
a_{i}^{T} p_{k}=\left(a_{i}^{T} x_{k}-b_{i}\right)^{\frac{1}{2}}\left(\hat{p}_{k}\right)_{i}=-\frac{\left(a_{i}^{T} x_{k}-b_{i}\right)\left(\lambda_{k+1}^{p}\right)_{i}}{\nu_{k}+\left|\left(\lambda_{k}\right)_{i}\right|} .
$$

Hence, there exists $1 \leq j \leq m$ such that,

$$
\beta_{k} \geq \frac{\nu_{k}+\left|\left(\lambda_{k}\right)_{j}\right|}{\left(\lambda_{k+1}^{p}\right)_{j}} \geq \frac{\nu_{k}}{\left\|\lambda_{k+1}^{p}\right\|_{\infty}} .
$$

From (2.6),

$$
\left[\begin{array}{c}
A^{T} \\
-D_{k}^{\frac{1}{2}}
\end{array}\right] \lambda_{k+1}^{p}=\left[\begin{array}{c}
\nabla f_{k} \\
0
\end{array}\right]+\left(\hat{H}_{k}+\nu_{k} I\right)\left(p_{k} ; \hat{p}_{k}\right) .
$$

Since $\left[A,-D_{k}^{\frac{1}{2}}\right]$ has full row rank in the compact set $\mathcal{L},\left\{\lambda_{k}\right\}$ is bounded (using Lemma 3.1 ), and $f(x)$ is twice continuously differentiable, there exist $\chi_{0}, \chi_{1}>0$ such that

$$
\left\|\lambda_{k+1}^{p}\right\|_{\infty} \leq \chi_{0}\left(\chi_{1}+\left(\chi_{2}+\nu_{k}\right) \Delta_{k}\right)
$$

Substitute (3.19) into (3.17), (3.16) holds.

Assume now that $\left\{x_{k}\right\}$ converges to $x_{*}$ satisfying strict complementarity, dual feasibility, and $\left\{\left(p_{k} ; \hat{p}_{k}\right)\right\}$ converges to zero. Since (AS.0) holds, $\left\{\lambda_{k}\right\}$ converges to $\lambda_{*}$. 
From strict complementarity and dual feasibility, $\left(\lambda_{*}\right)_{i}>0$ for any $a_{i}^{T} x_{*}-b_{i}=0$. Since $\left\{p_{k}\right\}$ converges to zero, the index $j$ defining $\beta_{k}$ in (3.17) satisfies $a_{j}^{T} x_{*}-b_{j}=0$ and $\left(\lambda_{*}\right)_{j}>0$ for sufficiently large $k$.

Let the sequence $\left\{\Delta_{k}\right\}$ be partitioned into two subsequences: $\left\{k: \Delta_{k} \leq 1\right\}$ and $\left\{k: \Delta_{k}>1\right\}$. For the subsequence $\left\{k: \Delta_{k} \leq 1\right\}$, using (3.17) and (3.19),

$$
\beta_{k} \geq \frac{\nu_{k}+\left|\left(\lambda_{k}\right)_{j}\right|}{\chi_{0}\left(\chi_{1}+\left(\chi_{2}+\nu_{k}\right)\right)}, \quad \text { for some } a_{j}^{T} x_{*}-b_{j}=0 \text {. }
$$

Since $\lim _{k \rightarrow \infty}\left(\lambda_{k}\right)_{i}>0$ for all $a_{i}^{T} x_{*}-b_{i}=0, \liminf _{k \rightarrow \infty} \beta_{k}>0$.

Consider the subsequence $\left\{k: \Delta_{k}>1\right\}$. Using Lemma 3.9,

$$
-\left(\psi_{k}\left(p_{k}^{*}\right)+\frac{1}{2} p_{k}^{* T} A^{T} D_{k}^{-1} C_{k} A p_{k}^{*}\right) \geq \frac{\beta_{q} \theta_{0}^{2} \min \left\{1, \beta_{k}^{2}\right\}}{2} \nu_{k} \Delta_{k}^{2} .
$$

Since $\left\{\left(p_{k}, \hat{p}_{k}\right)\right\}$ converges to zero, $\lim _{k \rightarrow \infty}\left(\psi_{k}\left(p_{k}^{*}\right)+\frac{1}{2} p_{k}^{* T} A^{T} D_{k}^{-1} C_{k} A p_{k}^{*}\right)=0$. Therefore $\lim _{k \rightarrow \infty} \nu_{k}=0$ for the subsequence $\left\{k: \Delta_{k}>1\right\}$. Using (3.18) and $\lim _{k \rightarrow \infty} \lambda_{k}=$ $\lambda_{*}, \lim _{k \rightarrow \infty} \lambda_{k}^{p}=\lambda_{*}$. Using (3.17), $\liminf _{k \rightarrow \infty} \beta_{k}>0$. The proof is completed.

Lemma 3.11. Assume that $\left[A, D_{*}\right]$ has full row rank at $x_{*} \in \mathcal{F}$ and the complementarity conditions are satisfied at $\left(x_{*} ; \lambda_{*}\right)$. Let the columns of $Z_{*}$ denote an orthonormal basis for the null space of $\left[A, D_{*}\right]$. If $Z_{*}^{T} \hat{H}_{*} Z_{*}$ is positive semidefinite, then $d^{T} \nabla^{2} f_{*} d \geq 0$ for any $d$ satisfying $a_{i}^{T} d=0$ for all $i$ with $a_{i}^{T} x_{*}-b_{i}=0$.

Proof. Let $d$ satisfy $a_{i}^{T} d=0$ for all $i$ with $a_{i}^{T} x_{*}-b_{i}=0$. Define $\hat{d}_{i}=\left(a_{i}^{T} x_{*}-\right.$ $\left.b_{i}\right)^{-\frac{1}{2}} a_{i}^{T} d$ if $a_{i}^{T} x_{*}-b_{i}>0$ and $\hat{d}_{i}=0$ otherwise. Then $A d-D_{*} \hat{d}=0$. Hence $(d ; \hat{d})=Z_{*} w$ and $\hat{d}^{T} C_{*} \hat{d}=0$. Since $Z_{*}^{T} \hat{H}_{*} Z_{*}$ is positive semidefinite, $d^{T} \nabla^{2} f_{*} d=$ $w^{T} Z_{*}^{T} \hat{H}_{*} Z_{*} w \geq 0$.

The next theorem establishes that the second order necessary conditions can be satisfied.

Theorem 3.12. Assume (AS.O) holds and $f(x): \mathcal{F} \rightarrow \Re$ is twice continuously differentiable. Let $\left\{x_{k}\right\}$ be the sequence generated by TRAM. If (AS.1) is satisfied at every iteration and (AS.3) holds for sufficiently large $k$, then

(a) There is a limit point $x_{*}$ such that $d^{T} \nabla^{2} f_{*} d \geq 0$ for any $d$ satisfying $a_{i}^{T} d=0$ for all $i$ with $a_{i}^{T} x_{*}-b_{i}=0$;

(b) If $x_{*}$ is an isolated limit point, then $d^{T} \nabla^{2} f_{*} d \geq 0$ for any $d$ satisfying $a_{i}^{T} d=0$ for all $i$ with $a_{i}^{T} x_{*}-b_{i}=0$.

Proof. Using Lemma 3.11, in order to prove $d^{T} \nabla^{2} f_{*} d \geq 0$ for any d satisfying $a_{i}^{T} d=0$ for all $i$ with $a_{i}^{T} x_{*}-b_{i}=0$, we only need to show that the projected Hessian $Z_{*}^{T} \hat{H}_{*} Z_{*}$ is positive semidefinite.

(a) If zero is a limit point of $\left\{\nu_{k}\right\}$, the result immediately follows. We prove that zero is a limit point of $\left\{\nu_{k}\right\}$ by contradiction. Assume that $\nu_{k} \geq \epsilon>0$ for sufficiently large $k$. First we show that this implies that $\left\{x_{k}\right\}$ converges and $\left\{\Delta_{k}\right\}$ converges to zero.

Using Lemma 3.9 for sufficiently large $k$,

$$
-\left(\psi_{k}\left(s_{k}\right)+\frac{1}{2} s_{k}^{T} A^{T} D_{k}^{-1} C_{k} A s_{k}\right) \geq \frac{\beta_{q} \theta_{0}^{2}}{2} \min \left\{1, \beta_{k}^{2}\right\} \epsilon \Delta_{k}^{2} .
$$


Hence, for sufficiently large $k$ and a successful step,

$$
f\left(x_{k}\right)-f\left(x_{k+1}\right) \geq \frac{\beta_{q} \theta_{0}^{2} \mu}{2} \min \left\{1, \beta_{k}^{2}\right\} \epsilon \Delta_{k}^{2} .
$$

If there are finite number of successful steps, $\left\{x_{k}\right\}$ converges and $\left\{\Delta_{k}\right\}$ converges to zero. Otherwise, let $\left\{k_{i}\right\}$ be the infinite sequence of successful iterations. Using Lemma 3.10,

$$
\beta_{k} \geq \frac{\nu_{k}}{\chi_{0}\left(\chi_{1}+\left(\chi_{2}+\nu_{k}\right) \Delta_{k}\right)} .
$$

Inequality (3.21), (3.22) and $\left\{f\left(x_{k}\right)-f\left(x_{k+1}\right)\right\}$ converges to zero imply that $\left\{\Delta_{k}\right\}$ is bounded, i.e., there exists $\chi_{\Delta}>0$ such that $\Delta_{k} \leq \chi_{\Delta}$. Hence

$$
\beta_{k} \geq \frac{\nu_{k}}{\chi_{0}\left(\chi_{1}+\left(\chi_{2}+\nu_{k}\right) \Delta_{k}\right)} \geq \frac{1}{\chi_{0}\left(\frac{\chi_{1}}{\epsilon}+\left(\frac{\chi_{2}}{\epsilon}+1\right) \chi_{\Delta}\right)}
$$

This inequality, (3.21), and $\left\{f\left(x_{k}\right)-f\left(x_{k+1}\right)\right\}$ converging to zero imply that

$$
\sum_{i=1}^{\infty} \Delta_{k_{i}}^{2}<\infty
$$

The trust region updating rules of TRAM then imply that

$$
\sum_{k=1}^{\infty} \Delta_{k}^{2} \leq\left(1+\frac{\gamma_{2}^{2}}{1-\gamma_{1}}\right) \sum_{i=1}^{\infty} \Delta_{k_{i}}^{2}
$$

Hence $\left\{x_{k}\right\}$ converges and $\left\{\Delta_{k}\right\}$ converges to zero. Since $\left\|s_{k}\right\|_{2} \leq$ $\beta_{s} \Delta_{k}$ and $\left\|p_{k}\right\|_{2} \leq \Delta_{k}$, both $\left\{s_{k}\right\}$ and $\left\{p_{k}\right\}$ converge to zero.

From $(3.23),(3.20)$ and $-\psi_{k}\left(s_{k}\right) \geq-\left(\psi_{k}\left(s_{k}\right)+\frac{1}{2} s_{k}{ }^{T} A^{T} D_{k}^{-1} C_{k} A s_{k}\right)$, there exists $\chi_{3}>0$ such that

$$
-\psi_{k}\left(s_{k}\right) \geq \chi_{3} \Delta_{k}^{2} \geq \frac{\chi_{3}}{\beta_{s}^{2}}\left\|s_{k}\right\|_{2}^{2}
$$

A standard estimate is that

$$
\left|f\left(x_{k}+s_{k}\right)-f\left(x_{k}\right)\right| \leq\left\|s_{k}\right\|_{2}^{2} \max _{0 \leq \xi_{k} \leq 1}\left\|\nabla^{2} f\left(x_{k}+\xi_{k} s_{k}\right)-\nabla^{2} f\left(x_{k}\right)\right\|_{2} .
$$

The last two inequalities, convergence of $\left\{x_{k}\right\}$ and $\left\{s_{k}\right\}$ converging to zero imply that $\left\{\left|\rho_{k}-1\right|\right\}$ converges to zero. Therefore the entire sequence $\left\{\rho_{k}\right\}$ converges to unity. The trust region updating rules of TRAM imply that $\left\{\Delta_{k}\right\}$ cannot converge to zero, which is a contradiction. Hence there is a limit point with $Z_{*}^{T} \hat{H}_{*} Z_{*}$ positive semidefinite. 
(b) If $\left\{x_{k}\right\}$ converges to $x_{*}$, the result follows from (a). If $\left\{x_{k}\right\}$ does not converge then Lemma 3.8 applies. Thus, if $\left\{x_{l_{j}}\right\}$ is the subsequence guaranteed by Lemma 3.8 , then $\Delta_{l_{j}} \geq \frac{1}{\beta_{s}} \epsilon$. From Lemma 3.9,

$$
-\left(\psi_{k}\left(s_{l_{j}}\right)+\frac{1}{2} s_{l_{j}}{ }^{T} A^{T} D_{l_{j}}^{-1} C_{l_{j}} A s_{l_{j}}\right) \geq \frac{\beta_{q} \theta_{0}^{2} \min \left\{1, \beta_{k}^{2}\right\}}{2} \nu_{l_{j}} \Delta_{l_{j}}^{2} .
$$

Using Lemma 3.10, there exist $\chi_{0}, \chi_{1}, \chi_{2}>0$ such that, for sufficiently large $j$,

$$
\beta_{l_{j}} \geq \frac{\nu_{l_{j}}}{\chi_{0}\left(\chi_{1}+\left(\chi_{2}+\nu_{k}\right) \Delta_{l_{j}}\right)}
$$

Hence

$$
f\left(x_{l_{j}}\right)-f\left(x_{l_{j}+1}\right) \geq \frac{\mu \beta_{q} \theta_{0}^{2}}{2} \min \left\{1,\left(\frac{\nu_{l_{j}}}{\chi_{0}\left(\chi_{1}+\left(\chi_{2}+\nu_{l_{j}}\right) \Delta_{l_{j}}\right)}\right)^{2}\right\} \nu_{l_{j}} \Delta_{l_{j}}^{2} .
$$

From $\lim _{j \rightarrow \infty} f\left(x_{l_{j}}\right)-f\left(x_{l_{j}+1}\right)=0$ and $\Delta_{l_{j}} \geq \frac{1}{\beta_{s}} \epsilon$, the above inequality implies that $\left\{\nu_{l_{j}}\right\}$ converges to zero. Thus $Z_{*}^{T} \hat{H}_{*} Z_{*}$ is positive semidefinite.

We have established (a)-(b).

4. Local Convergence. Let $F(x, \lambda)=0$ denote the complementarity conditions, i.e.,

$$
F(x, \lambda) \stackrel{\text { def }}{=}\left[\begin{array}{l}
\nabla f(x)-A^{T} \lambda \\
D(x) \lambda
\end{array}\right],
$$

where $D(x)=\operatorname{diag}(A x-b)$ as in (2.1). Then the Jacobian matrix $J(x, \lambda)$ of $F(x, \lambda)$ is

$$
J(x, \lambda)^{T} \stackrel{\text { def }}{=}\left[\begin{array}{cc}
\nabla^{2} f(x) & -A^{T} \\
\operatorname{diag}(\lambda) A & D(x)
\end{array}\right] .
$$

From (2.4), a Newton step $p_{k}^{N}$ of the trust region subproblem (2.5) is an approximate Newton step for the complementarity conditions $F(x, \lambda)=0$.

To establish the quadratic convergence of the proposed TRAM, we prove that, asymptotically, the Newton step $p_{k}^{N}$ of the trust region subproblem is a sufficiently accurate approximation to the exact Newton step of $F(x, \lambda)=0$. Moreover, asymptotically, the Newton step $p_{k}^{N}$ is in the interior of the trust region (hence $p_{k}=p_{k}^{N}$ ) and (AS.1), (AS.2) and (AS.3) can be satisfied by a damped Newton step $p_{k}^{N}$.

First, we prove that the Jacobian matrix $J(x, \lambda)$ is nonsingular near a local minimizer satisfying strict complementarity and linear independence assumptions.

THEOREM 4.1. Assume that $f(x): \mathcal{F} \rightarrow \Re$ is twice continuously differentiable and the second order sufficiency conditions of (1.1) are satisfied at $\left(x_{*} ; \lambda_{*}\right)$. Assume further that strict complementarity holds at $x_{*}$ and $\left\{a_{i}: a_{i}^{T} x_{*}-b_{i}=0\right\}$ are linearly independent. Then

(a) The Jacobian matrix $J\left(x_{*}, \lambda_{*}\right)$ is nonsingular; 
(b) The symmetric matrix $A^{T}\left(\operatorname{diag}(|\lambda|) D(x)^{-1}\right) A+\nabla^{2} f(x)$ is positive definite when $x \in \operatorname{int}(\mathcal{F})$ and $(x ; \lambda)$ is sufficiently close to $\left(x_{*} ; \lambda_{*}\right)$.

Proof. Assume that there exists $(v ; w), v \in \Re^{n}$ and $w \in \Re^{m}$, such that

$$
\left[\begin{array}{ll}
\nabla^{2} f_{*} & -A^{T} \\
\operatorname{diag}\left(\lambda_{*}\right) A & D_{*}
\end{array}\right]\left[\begin{array}{l}
v \\
w
\end{array}\right]=0 .
$$

Without loss of generality, partition $A=\left[A_{0} ; A_{1}\right]$ where the rows of $A_{0}$ correspond to the indices $\left\{i: a_{i}^{T} x_{*}-b_{i}=0\right\}$ and the rows of $A_{1}$ correspond to the indices $\left\{i: a_{i}^{T} x_{*}-b_{i} \neq 0\right\}$. Since strict complementarity holds at $\left(x_{*} ; \lambda_{*}\right), A_{0} v=0, w_{1}=$ 0 , and $\nabla^{2} f_{*} v-A_{0}^{T} w_{0}=0$. Hence $v^{T} \nabla^{2} f_{*} v=0$. Since the second order sufficiency conditions are satisfied at $\left(x_{*} ; \lambda_{*}\right)$ and $A_{0} v=0, v=0$ holds. This implies that $A^{T} w=0$. Therefore $A_{0}^{T} w_{0}=0$. Applying linear independence assumption of the columns of $A_{0}^{T}, w=0$. Hence the Jacobian matrix $J\left(x_{*}, \lambda_{*}\right)$ is nonsingular.

Without loss of generality, assume that $\|u+v\|_{2}=1 u, v \in \Re^{n}$, with $u^{T} v=0$ and $A_{0} v=0$. Since the second order sufficiency conditions are satisfied at $\left(x_{*} ; \lambda_{*}\right)$ with strict complementarity, there exist $\chi_{0}, \sigma_{\min }, \sigma_{\max }>0$, such that in a neighborhood of $\left(x_{*} ; \lambda_{*}\right)$,

$$
\min \left(\lambda_{0}\right) \geq \chi_{0}, \quad v^{T} \nabla^{2} f(x) v \geq \sigma_{\min }\|v\|_{2}^{2}, \quad \text { and } \quad\left\|\nabla^{2} f(x)\right\|_{2} \leq \sigma_{\max } .
$$

Using $\|v\|_{2}^{2}=1-\|u\|_{2}^{2},\|v\|_{2} \leq 1$ and $\|u\|_{2} \leq 1$,

$$
\begin{aligned}
(u+v)^{T} \nabla^{2} f(x)(u+v) & =v^{T} \nabla^{2} f(x) v+2 u^{T} \nabla^{2} f(x) v+u^{T} \nabla^{2} f(x) u \\
& \geq \sigma_{\min }\|v\|_{2}^{2}-2 \sigma_{\max }\|u\|_{2}\|v\|_{2}-\sigma_{\max }\|u\|_{2}^{2} \\
& =\sigma_{\min }\|v\|_{2}^{2}-\sigma_{\max }\|u\|_{2}\left(2\|v\|_{2}+\|u\|_{2}\right) \\
& \geq \sigma_{\min }-\left(\sigma_{\min }+3 \sigma_{\max }\right)\|u\|_{2}
\end{aligned}
$$

Let $M=A^{T}\left(\operatorname{diag}(|\lambda|) D(x)^{-1}\right) A+\nabla^{2} f(x)$. From the above inequality and (4.1),

$$
\begin{aligned}
& (u+v)^{T} M(u+v) \\
\geq & v^{T} \nabla^{2} f(x) v+2 u^{T} \nabla^{2} f(x) v+u^{T} \nabla^{2} f(x) u+u^{T} A_{0}^{T} D_{0}^{-1} \operatorname{diag}\left(\lambda_{0}\right) A_{0} u \\
\geq & \sigma_{\min }-\left(\sigma_{\min }+3 \sigma_{\max }\right)\|u\|_{2}+\frac{\chi_{0}}{\left\|A_{0} x-b_{0}\right\|_{\infty}}\left\|A_{0} u\right\|_{2}^{2} .
\end{aligned}
$$

If $\|u\|_{2}<\chi_{1} \stackrel{\text { def }}{=} \frac{\sigma_{\min }}{2\left(\sigma_{\min }+3 \sigma_{\max }\right)}$, then $(u+v)^{T} M(u+v) \geq \frac{1}{2} \sigma_{\min }$. If $\|u\|_{2} \geq \chi_{1}$, there exists $\chi_{2}>0$ such that $\left\|A_{0} u\right\|_{2} \geq \chi_{2}\|u\|_{2}^{2} \geq \chi_{1}^{2} \chi_{2}$ since $A_{0}$ is of full row rank. Hence

$$
(u+v)^{T} M(u+v) \geq \frac{\chi_{0} \chi_{1}^{2} \chi_{2}}{\left\|A_{0} x-b_{0}\right\|_{\infty}}-\left(\sigma_{\min }+3 \sigma_{\max }\right) .
$$

When $\left\|A_{0} x-b_{0}\right\|_{\infty}<\frac{2 \chi_{0} \chi_{1}^{2} \chi_{2}}{\sigma_{\min }+3 \sigma_{\max }},(u+v)^{T} M(u+v)>\sigma_{\min }+3 \sigma_{\max }$. Hence there exists a neighborhood of $\left(x_{*} ; \lambda_{*}\right)$ in which the symmetric matrix $M$ is positive definite. The proof is completed. $\square$

We have established in Theorem 3.7 and 3.12 that, assuming sufficient decrease and strict complementarity, there exists a limit point satisfying the first order and second order optimality conditions. We prove next that, assuming the second order 
sufficiency assumption at this limit, convergence occurs and trust region size $\left\{\Delta_{k}\right\}$ is asymptotically bounded away from zero.

Theorem 4.2. Assume that (AS.0) and (AS.1) hold and $f(x): \mathcal{F} \rightarrow \Re$ is twice continuously differentiable. Let $\left\{x_{k}\right\}$ be the sequence generated by TRAM. Assume further that (AS.3) holds for sufficiently large $k$ and the second order sufficiency conditions and strict complementarity are satisfied at a limit $x_{*}$ of $\left\{x_{k}\right\}$. Then $\left\{x_{k}\right\}$ converges to $x_{*}, Z_{k}^{T} \hat{H}_{k} Z_{k}$ is positive definite for sufficiently large $k$, all iterations are eventually successful, and $\left\{\Delta_{k}\right\}$ is bounded away from zero.

Proof. For any $w \in \Re^{m}$, let $(d ; \hat{d})=Z_{k}^{T} w$ where $d \in \Re^{n}$ and $\hat{d} \in \Re^{m}$. Then $A d-D_{k}^{\frac{1}{2}} \hat{d}=0$, i.e., $\hat{d}=D_{k}^{-\frac{1}{2}} A d$. Let $\left.M_{k}=A^{T}\left(\operatorname{diag}\left(\left|\lambda_{k}\right|\right) D_{k}\right)^{-1}\right) A+\nabla^{2} f_{k}$. Using Theorem 4.1 and $C_{k}=\operatorname{diag}\left(\lambda_{k}\right)$,

$$
w^{T} Z_{k}^{T} \hat{H}_{k} Z_{k} w=(d ; \hat{d})^{T} \hat{H}_{k}(d ; \hat{d})=d^{T} M_{k} d>0 \text {, if } w \neq 0 .
$$

Hence $Z_{k}^{T} \hat{H}_{k} Z_{k}$ is positive definite when $\left(x_{k} ; \lambda_{k}\right)$ is sufficiently close to $\left(x_{*} ; \lambda_{*}\right)$.

Since the second order sufficiency conditions are satisfied at $x_{*}$, the point $x_{*}$ is an isolated limit point. Let $\hat{s}_{k}=D_{k}^{-\frac{1}{2}} A s_{k}$. Then $Z_{k} Z_{k}^{T}\left(s_{k} ; \hat{s}_{k}\right)=\left(s_{k} ; \hat{s}_{k}\right)$. Since (AS.3) is satisfied for sufficiently large $k, \psi_{k}\left(s_{k}\right)+\frac{1}{2} s_{k}{ }^{T} A^{T} D_{k}^{-1} C_{k} A s_{k}=\nabla f_{k}^{T} s_{k}+$ $\frac{1}{2}\left(s_{k} ; \hat{s}_{k}\right)^{T} \hat{H}_{k}\left(s_{k} ; \hat{s}_{k}\right)<0$ asymptotically. Hence

$$
\begin{aligned}
0 \leq \frac{1}{2}\left(s_{k} ; \hat{s}_{k}\right)^{T} Z_{k}\left(Z_{k}^{T} \hat{H}_{k} Z_{k}\right) Z_{k}^{T}\left(s_{k} ; \hat{s}_{k}\right) & <-\left[\nabla f_{k}, 0\right]^{T} Z_{k} Z_{k}^{T}\left(s_{k} ; \hat{s}_{k}\right) \\
& \leq\left\|Z_{k}^{T}\left(s_{k} ; \hat{s}_{k}\right)\right\|_{2}\left\|Z_{k}^{T}\left(\nabla f_{k} ; 0\right)\right\|_{2},
\end{aligned}
$$

whenever $Z_{k}^{T} \hat{H}_{k} Z_{k}$ is positive definite and $k$ is sufficiently large. But

$$
\left(s_{k} ; \hat{s}_{k}\right)^{T} Z_{k}\left(Z_{k}^{T} \hat{H}_{k} Z_{k}\right) Z_{k}^{T}\left(s_{k} ; \hat{s}_{k}\right) \geq \frac{1}{\left\|\left(Z_{k}^{T} \hat{H}_{k} Z_{k}\right)^{-1}\right\|_{2}}\left\|Z_{k}^{T}\left(s_{k} ; \hat{s}_{k}\right)\right\|_{2}^{2} .
$$

This implies that, for sufficiently large $k$,

$$
\frac{1}{2}\left\|Z_{k}^{T}\left(s_{k} ; \hat{s}_{k}\right)\right\|_{2} \leq\left\|\left(Z_{k}^{T} \hat{H}_{k} Z_{k}\right)^{-1}\right\|_{2}\left\|Z_{k}^{T}\left(\nabla f_{k} ; 0\right)\right\|_{2},
$$

whenever $Z_{k}^{T} \hat{H}_{k} Z_{k}$ is positive definite. From Theorem 3.7 and

$$
\left\|Z_{k} Z_{k}^{T}\left(\nabla f_{k} ; 0\right)\right\|_{2}^{2}=-\nabla f_{k}^{T} g_{k}
$$

$\left\{Z_{k}^{T}\left(\nabla f_{k} ; 0\right)\right\}$ converges to zero. Hence $\left\{\left(p_{k} ; \hat{p}_{k}\right)\right\}$ and $\left\{s_{k}\right\}$ converge to zero. Following Lemma $3.8,\left\{x_{k}\right\}$ converges to $x_{*}$.

Let $\kappa$ be an upper bound on the condition number of $Z_{k}^{T} \hat{H}_{k} Z_{k}$. From $Z_{k}^{T}\left(\nabla f_{k} ; 0\right)=$ $-\left(Z_{k}^{T} \hat{H}_{k} Z_{k}\right)^{T}\left(p_{k}^{N} ; \hat{p}_{k}^{N}\right)$ and $(4.2)$,

$$
\frac{1}{2}\left\|\left(s_{k} ; \hat{s}_{k}\right)\right\|_{2} \leq \kappa\left\|\left(p_{k}^{N} ; \hat{p}_{k}^{N}\right)\right\|_{2}
$$

whenever $\left\{Z_{k}^{T} \hat{H}_{k} Z_{k}\right\}$ is positive definite and $k$ is sufficiently large.

Since $\left\{x_{k}\right\}$ converges to $x_{*}$ satisfying the second order sufficiency conditions with strict complementarity and $\left\{\left(p_{k} ; \hat{p}_{k}\right)\right\}$ converges to zero, using Lemma 3.9 and 3.10, there exists $\chi_{0}>0$ such that, for sufficiently large $k$,

$$
-\left(\psi_{k}\left(p_{k}^{*}\right)+\frac{1}{2} p_{k}^{* T} A^{T} D_{k}^{-1} C_{k} A p_{k}^{*}\right) \geq \frac{\beta_{q} \theta_{0}^{2} \chi_{0}}{2}\left\|R_{k} Z_{k}^{T}\left(p_{k} ; \hat{p}_{k}\right)\right\|_{2}^{2} .
$$


Since the eigenvalues of $\left\{Z_{k}^{T} \hat{H}_{k} Z_{k}\right\}$ are positive and bounded away from zero asymptotically, there exists $\chi_{1}>0$ such that, for sufficiently large $k$,

$$
-\left(\psi_{k}\left(p_{k}^{*}\right)+\frac{1}{2} p_{k}^{* T} A^{T} D_{k}^{-1} C_{k} A p_{k}^{*}\right) \geq \chi_{1} \min \left\{\Delta_{k}^{2},\left\|\left(p_{k}^{N} ; \hat{p}_{k}^{N}\right)\right\|_{2}^{2}\right\} .
$$

Using (4.3) and (AS.3), there exists $\chi_{2}>0$ such that

$$
-\left(\psi_{k}\left(s_{k}\right)+\frac{1}{2} s_{k}^{T} A^{T} D_{k}^{-1} C_{k} A s_{k}\right) \geq \chi_{2}\left\|\left(s_{k} ; \hat{s}_{k}\right)\right\|_{2}^{2} \geq \chi_{2}\left\|s_{k}\right\|_{2}^{2}
$$

Therefore $-\psi_{k}\left(s_{k}\right) \geq \chi_{2}\left\|s_{k}\right\|_{2}^{2}$ for sufficiently large $k$. But $f(x)$ is twice continuously differentiable on $\mathcal{F}$,

$$
\begin{aligned}
& \left|f\left(x_{k}+s_{k}\right)-f\left(x_{k}\right)-\psi_{k}\left(s_{k}\right)\right| \\
\leq & \left\|s_{k}\right\|_{2}^{2} \max _{0 \leq \xi_{k} \leq 1}\left\|\nabla^{2} f\left(x_{k}+\xi_{k} s_{k}\right)-\nabla^{2} f\left(x_{k}\right)\right\|_{2} .
\end{aligned}
$$

Since $\left\{x_{k}\right\}$ converges and $\left\{s_{k}\right\}$ converges to zero, using (4.4), $\lim _{k \rightarrow \infty} \rho_{k}=1$. This implies that $\rho_{k}>\eta$ for $k$ sufficiently large. Therefore $\left\{\Delta_{k}\right\}$ is bounded away from zero. $\mathrm{c}$

Recall that $p_{k}^{N}$ denotes the Newton step of the trust region subproblem and satisfies (2.4). Lemma 4.3 shows that the stepsize $\alpha_{k}^{*}$ converges to one.

Lemma 4.3. Assume that $f(x): \mathcal{F} \rightarrow \Re$ is twice continuously differentiable and $\left\{x_{k}\right\}$ converges to $x_{*}$. Moreover, $\left\{a_{i}: a_{i}^{T} x_{*}-b_{i}=0\right\}$ are linearly independent and the second order sufficiency conditions and strict complementarity are satisfied at $x_{*}$. Then, both $\left\{\lambda_{k}\right\}$ and $\left\{\lambda_{k}^{p}\right\}$ converge to $\lambda_{*}$ and

$$
\begin{aligned}
& \left\|\lambda_{k}-\lambda_{*}\right\|_{2}=O\left(\left\|x_{k}-x_{*}\right\|_{2}\right), \\
& \left\|\lambda_{k}-\lambda_{k}^{p}\right\|_{2}=O\left(\left\|x_{k}-x_{*}\right\|_{2}+\left\|\lambda_{k}^{p}-\lambda_{*}\right\|_{2}\right), \\
& \left\|\lambda_{k+1}^{p}-\lambda_{k}\right\|_{2}=O\left(\left\|\left(x_{k} ; \lambda_{k}^{p}\right)-\left(x_{*} ; \lambda_{*}\right)\right\|_{2}\right), \\
& \left\|\alpha_{k}^{*}-1\right\|_{2}=O\left(\left\|\left(x_{k} ; \lambda_{k}^{p}\right)-\left(x_{*} ; \lambda_{*}\right)\right\|_{2}\right),
\end{aligned}
$$

where $\alpha_{k}^{*}=\min \left\{1, \beta_{k}\right\}$ and $\beta_{k}$ is the stepsize to the boundary of the feasible region $\mathcal{F}$ along $p_{k}^{N}$.

Proof. By definition (2.12) of $\lambda_{k},\left(A A^{T}+D_{k}^{2}\right) \lambda_{k}=A \nabla f_{k}$. Since $\left\{a_{i}: a_{i}^{T} x_{*}-\right.$ $\left.b_{i}=0\right\}$ are linearly independent, $\left[A,-D_{*}\right]$ has full row rank and $\left(A A^{T}+D_{*}^{2}\right)$ is nonsingular. But $\nabla f(x)$ is continuous in $\mathcal{F}$. Thus $\left\|\lambda_{k}-\lambda_{*}\right\|_{2}=O\left(\left\|x_{k}-x_{*}\right\|_{2}\right)$. Since the coefficient matrix $(2.4)$ is equivalent to $J(x, \lambda)^{T}$ at $\left(x_{*} ; \lambda_{*}\right)$, applying Theorem 4.1, the linear system (2.4) is nonsingular at $\left(x_{*} ; \lambda_{*}\right)$. Since $\left\{x_{k}\right\}$ converges to $x_{*}$ and complementarity is satisfied at $x_{*},\left\{D_{k} \lambda_{k}\right\}$ and $\left\{\nabla f_{k}-A^{T} \lambda_{k}\right\}$ converge to zero. From (2.4) and $\left\{\lambda_{k}\right\}$ converges to $\lambda_{*},\left\{\lambda_{k}^{p}\right\}$ converges to $\lambda_{*}$ and $\lim _{k \rightarrow \infty} p_{k}^{N}=0$. Moreover, $\left\|\lambda_{k}-\lambda_{k}^{p}\right\|_{2} \leq\left\|\lambda_{k}-\lambda_{*}\right\|_{2}+\left\|\lambda_{k}^{p}-\lambda_{*}\right\|_{2}=O\left(\left\|x_{k}-x_{*}\right\|_{2}+\left\|\lambda_{k}^{p}-\lambda_{*}\right\|_{2}\right)$. From (2.4),

$$
\begin{aligned}
\left\|\lambda_{k+1}^{p}-\lambda_{k}\right\|_{2} & =O\left(\left\|\left(x_{k} ; \lambda_{k}\right)-\left(x_{*} ; \lambda_{*}\right)\right\|_{2}\right) \\
& =O\left(\left\|\left(x_{k} ; \lambda_{k}^{p}\right)-\left(x_{*} ; \lambda_{*}\right)\right\|_{2}\right)+O\left(\left\|\lambda_{k}^{p}-\lambda_{k}\right\|_{2}\right) \\
& =O\left(\left\|\left(x_{k} ; \lambda_{k}^{p}\right)-\left(x_{*} ; \lambda_{*}\right)\right\|_{2}\right) .
\end{aligned}
$$


If $\beta_{k}=\infty, \alpha_{k}^{*}-1=0$. Consider the case when $\beta_{k}<\infty$. Using (2.4) again, for $1 \leq i \leq m$,

$$
a_{i}^{T} p_{k}^{N}=-\frac{\left(a_{i}^{T} x_{k}-b_{i}\right)\left(\lambda_{k+1}^{p}\right)_{i}}{\left|\left(\lambda_{k}\right)_{i}\right|} .
$$

Since $\left\{p_{k}^{N}\right\}$ converges to zero and strict complementarity is satisfied at $x_{*}$, for sufficiently large $k$,

$$
\beta_{k}=\frac{\left|\left(\lambda_{k}\right)_{j}\right|}{\left(\lambda_{k+1}^{p}\right)_{j}}, \text { for some }\left(\lambda_{k+1}^{p}\right)_{j}>0 \text { and } a_{j}^{T} x_{*}-b_{j}=0 .
$$

From strict complementarity, $\lim _{k \rightarrow \infty}\left(\lambda_{k}^{p}\right)_{j}=\left(\lambda_{*}\right)_{j}>0$ for any $a_{j}^{T} x_{*}-b_{j}=0$. Hence

$$
\left|1-\alpha_{k}^{*}\right|=O\left(\left|1-\beta_{k}\right|\right)=O\left(\left\|\lambda_{k+1}^{p}-\lambda_{k}\right\|_{2}\right)=O\left(\left\|\left(x_{k} ; \lambda_{k}^{p}\right)-\left(x_{*} ; \lambda_{*}\right)\right\|_{2}\right) .
$$

The proof is completed.

TheOREm 4.4. Assume $0<\mu<1$ and $f(x): \mathcal{F} \rightarrow \Re$ is twice continuously differentiable on $\mathcal{F}$. Assume that $\left\{x_{k}\right\}$ converges to $x_{*}$, the second order sufficiency conditions and strict complementarity are satisfied at $\left(x_{*} ; \lambda_{*}\right)$ and $\left\{a_{i}: a_{i}^{T} x_{*}-b_{i}=\right.$ $0\}$ are linearly independent. Moreover, $\left|\theta_{k}-1\right|=O\left(\left\|\left(x_{k} ; \lambda_{k}\right)-\left(x_{*} ; \lambda_{*}\right)\right\|_{2}\right)$. Then $\left|1-\alpha_{k}\right|=O\left(\left\|\left(x_{k} ; \lambda_{k}\right)-\left(x_{*} ; \lambda_{*}\right)\right\|\right)$, and

$$
f\left(x_{k}+\alpha_{k} p_{k}^{N}\right)-f\left(x_{k}\right)<\mu \psi_{k}\left(\alpha_{k} p_{k}^{N}\right), \quad \text { where } \alpha_{k}=\theta_{k} \alpha_{k}^{*}, \quad 0<\theta_{0} \leq \theta_{k}<1,
$$

$\alpha_{k}^{*}=\min \left\{1, \beta_{k}\right\}$ and $\beta_{k}$ is the stepsize to the boundary of the feasible region $\mathcal{F}$ along $p_{k}^{N}$. In addition, if $x_{k+1}=x_{k}+\alpha_{k} p_{k}^{N}$ for sufficiently large $k$ then $\left\{\left(x_{k} ; \lambda_{k}^{p}\right)\right\}$ converges quadratically to $\left(x_{*} ; \lambda_{*}\right)$.

Proof. Let $d_{k}=\alpha_{k} p_{k}^{N}$. Since $f(x)$ is twice continuously differentiable in $\mathcal{F}$, there exists $0 \leq \xi_{k} \leq 1$ such that

$$
f\left(x_{k}+d_{k}\right)-f\left(x_{k}\right)=\psi_{k}\left(d_{k}\right)+\frac{1}{2} d_{k}^{T}\left(\nabla^{2} f\left(x_{k}+\xi_{k} d_{k}\right)-\nabla^{2} f_{k}\right) d_{k} .
$$

Since $\left\{x_{k}\right\}$ converges to $x_{*}$ at which the second order sufficiency conditions are satisfied, using (2.4), $\left\{p_{k}^{N}\right\}$ and $\left\{d_{k}\right\}$ converge to zero. Therefore, there exists $\left\{\epsilon_{k}\right\}$ converging to zero such that, for sufficiently large $k$,

$$
f\left(x_{k}+d_{k}\right)-f\left(x_{k}\right)=\psi_{k}\left(d_{k}\right)+\epsilon_{k}\left\|d_{k}\right\|_{2}^{2} .
$$

From $0<\mu<1$,

$$
\begin{aligned}
& f\left(x_{k}+d_{k}\right)-f\left(x_{k}\right)-\mu \psi_{k}\left(d_{k}\right) \\
= & (1-\mu)\left(\nabla f_{k}^{T} d_{k}+\frac{1}{2} d_{k}^{T} \nabla^{2} f_{k} d_{k}\right)+\epsilon_{k}\left\|d_{k}\right\|_{2}^{2} \\
\leq & (1-\mu)\left(\nabla f_{k}^{T} d_{k}+\frac{1}{2}\left(d_{k} ; \hat{d}_{k}\right)^{T} \hat{H}_{k}\left(d_{k} ; \hat{d}_{k}\right)\right)+\epsilon_{k}\left\|d_{k}\right\|_{2}^{2} .
\end{aligned}
$$

Since the columns of $Z_{k}$ form an orthonormal basis for the null space of $\left[A,-D_{k}^{\frac{1}{2}}\right]$, and $A p_{k}^{N}-D_{k}^{\frac{1}{2}} \hat{p}_{k}^{N}=0,\left(p_{k}^{N} ; \hat{p}_{k}^{N}\right)=Z_{k} Z_{k}^{T}\left(p_{k} ; \hat{p}_{k}^{N}\right)$ and

$$
\begin{aligned}
\nabla f_{k}^{T} p_{k}^{N} & =-\left(p_{k}^{N} ; \hat{p}_{k}^{N}\right)^{T} \hat{H}_{k}\left(p_{k}^{N} ; \hat{p}_{k}^{N}\right) \\
& =-\left(p_{k}^{N} ; \hat{p}_{k}^{N}\right)^{T} Z_{k}\left(Z_{k}^{T} \hat{H}_{k} Z_{k}\right) Z_{k}^{T}\left(p_{k}^{N} ; \hat{p}_{k}^{N}\right) .
\end{aligned}
$$


Substitute $d_{k}=\alpha_{k} p_{k}^{N}$

$$
\alpha_{k} \nabla f_{k}^{T} d_{k}=-\left(d_{k} ; \hat{d}_{k}\right)^{T} Z_{k}\left(Z_{k}^{T} \hat{H}_{k} Z_{k}\right) Z_{k}^{T}\left(d_{k} ; \hat{d}_{k}\right) .
$$

Hence

$$
\begin{aligned}
& f\left(x_{k}+d_{k}\right)-f\left(x_{k}\right)-\mu \psi_{k}\left(d_{k}\right) \\
\leq & -\frac{2-\alpha_{k}}{2 \alpha_{k}}(1-\mu)\left(d_{k} ; \hat{d}_{k}\right)^{T} Z_{k}\left(Z_{k}^{T} \hat{H}_{k} Z_{k}\right) Z_{k}^{T}\left(d_{k} ; \hat{d}_{k}\right)+\epsilon_{k}\left\|d_{k}\right\|_{2}^{2} .
\end{aligned}
$$

From Theorem 4.2, the projected Hessian $Z_{k}^{T} \hat{H}_{k} Z_{k}$ is positive definite for sufficiently large $k$. Hence there exists $\epsilon>0$ such that, for $k$ sufficiently large,

$$
\left(d_{k} ; \hat{d}_{k}\right)^{T} Z_{k}\left(Z_{k}^{T} \hat{H}_{k} Z_{k}\right) Z_{k}^{T}\left(d_{k} ; \hat{d}_{k}\right) \geq \epsilon\left\|Z_{k}^{T}\left(d_{k} ; \hat{d}_{k}\right)\right\|_{2} .
$$

Hence $\left\|Z_{k}^{T}\left(d_{k} ; \hat{d}\right)\right\|_{2}^{2}=\left\|\left(d_{k} ; \hat{d}_{k}\right)\right\|_{2}^{2}$. Hence

$$
f\left(x_{k}+d_{k}\right)-f\left(x_{k}\right)-\mu \psi_{k}\left(d_{k}\right) \leq-\frac{2-\alpha_{k}}{2 \alpha_{k}}(1-\mu) \epsilon\left\|\left(d_{k} ; \hat{d}_{k}\right)\right\|_{2}^{2}+\epsilon_{k}\left\|d_{k}\right\|_{2}^{2} .
$$

From $\left\{\theta_{k}\right\}$ converges to $1,\left\{\alpha_{k}\right\}$ converges to 1 . But $\left\{\epsilon_{k}\right\}$ converges to zero. Hence, for sufficiently large $k$,

$$
f\left(x_{k}+d_{k}\right)-f\left(x_{k}\right)<\mu \psi_{k}\left(d_{k}\right) .
$$

Let $\lambda_{k+1}^{p}=\lambda_{k}^{p}+w_{k}$. Similar to $(2.4),\left(d_{k} ; w_{k}\right)$ satisfies

$$
\tilde{J}_{k}^{T}\left(d_{k} ; w_{k}\right)=-\left(\nabla f_{k}-A \lambda_{k}^{p} ; D_{k} \lambda_{k}^{p}\right)
$$

where

$$
\tilde{J}_{k}^{T}=\left[\begin{array}{ll}
\frac{1}{\alpha_{k}} \nabla^{2} f_{k} & -A^{T} \\
\frac{1}{\alpha_{k}} C_{k} A & D_{k}
\end{array}\right],
$$

and

$$
\left\|J\left(x_{k}, \lambda_{k}^{p}\right)^{T}-\tilde{J}_{k}^{T}\right\|_{2} \leq\left\|\left(\operatorname{diag}\left(\lambda_{k}^{p}\right)-C_{k}\right) A\right\|_{2}+\left|1-\frac{1}{\alpha_{k}}\right|\left\|\nabla^{2} f_{k}\right\|_{2}+\left|1-\frac{1}{\alpha_{k}}\right|\left\|C_{k} A\right\|_{2} .
$$

Using Lemma $4.3,\left|\alpha_{k}^{*}-1\right|=O\left(\left\|\left(x_{k} ; \lambda_{k}\right)-\left(x_{*} ; \lambda_{*}\right)\right\|_{2}\right)$. In addition, $\left|\theta_{k}-1\right|=$ $O\left(\left\|\left(x_{k} ; \lambda_{k}\right)-\left(x_{*} ; \lambda_{*}\right)\right\|_{2}\right)$ and $0<\left(1-\theta_{k} \alpha_{k}^{*}\right)<\left(1-\theta_{k}\right)+\theta_{k}\left(1-\alpha_{k}^{*}\right)$. Hence $\left|\alpha_{k}-1\right|=$ $O\left(\left\|\left(x_{k} ; \lambda_{k}\right)-\left(x_{*} ; \lambda_{*}\right)\right\|_{2}\right)$. Since the first order necessary conditions are satisfied at $\left(x_{*} ; \lambda_{*}\right)$ with strict complementarity, using Lemma 4.3,

$$
\left\|\operatorname{diag}\left(\lambda_{k}^{p}\right)-C_{k}\right\|_{2}=O\left(\left\|\left(x_{k} ; \lambda_{k}^{p}\right)-\left(x_{*} ; \lambda_{*}\right)\right\|_{2}\right) .
$$

Thus

$$
\left\|J\left(x_{k}, \lambda_{k}^{p}\right)^{T}-\tilde{J}_{k}^{T}\right\|_{2}=O\left(\left\|\left(x_{k} ; \lambda_{k}^{p}\right)-\left(x_{*} ; \lambda_{*}\right)\right\|_{2}\right) .
$$

Applying Theorem 3.4 in [11], $\left\{\left(x_{k} ; \lambda_{k}^{p}\right)\right\}$ converges quadratically to $\left(x_{*} ; \lambda_{*}\right)$.

TheOREM 4.5. Assume that (AS.O) holds and $f(x): \mathcal{F} \rightarrow \Re$ is twice continuously differentiable on $\mathcal{F}$. Assume (AS.1) is satisfied at every iteration, and (AS.2) 
and (AS.3) hold for sufficiently large $k$. Let $\left\{x_{k}\right\}$ be generated by TRAM in FIG. 1 and $x_{*}$ be a limit point. Assume further that the second order sufficiency conditions are satisfied at $\left(x_{*} ; \lambda_{*}\right)$ with strict complementarity, and $\left|\theta_{k}-1\right|=O\left(\left\|\left(x_{k} ; \lambda_{k}\right)-\left(x_{*} ; \lambda_{*}\right)\right\|_{2}\right)$. Let $\alpha_{k}=\theta_{k} \min \left\{1, \beta_{k}\right\}$ where $\beta_{k}$ is the stepsize along $p_{k}$ to the boundary of $\mathcal{F}$. Then, for sufficiently large $k, \alpha_{k} p_{k}$ satisfies (AS.1), (AS.2) and (AS.3). If $x_{k+1}=x_{k}+\alpha_{k} p_{k}$ for sufficiently large $k$, then $\left\{\left(x_{k} ; \lambda_{k}^{p}\right)\right\}$ converges to $\left(x_{*} ; \lambda_{*}\right)$ quadratically.

Proof. From Theorem 4.2, $\left\{x_{k}\right\}$ converges to $x_{*}$ and $\left\{\Delta_{k}\right\}$ is bounded away from zero. Since $p_{k}^{N}$ satisfies

$$
\left(Z_{k}^{T} \hat{H}_{k} Z_{k}\right) Z_{k}^{T}\left(p_{k}^{N} ; \hat{p}_{k}^{N}\right)=-Z_{k}^{T}\left(\nabla f_{k} ; 0\right),
$$

and $\left\{\nabla f_{k}^{T} g_{k}\right\}$ converges to zero, the sequence $\left\{\left(p_{k}^{N} ; \hat{p}_{k}^{N}\right)\right\}$ converges to zero. Hence, for sufficiently large $k,\left\|\left(p_{k}^{N} ; \hat{p}_{k}^{N}\right)\right\|_{2}<\Delta_{k}$ and $p_{k}=p_{k}^{N}$.

Using Theorem 4.4, $\left\{\alpha_{k}\right\}$ converges to 1 . From $0 \leq \alpha_{k} \leq 1$ and $\left(p_{k} ; \hat{p}_{k}\right) \hat{H}_{k}\left(p_{k}, \hat{p}_{k}\right) \geq$ 0 for sufficiently large $k$,

$$
\psi_{k}\left(\alpha_{k} p_{k}\right)+\frac{\alpha_{k}^{2}}{2} p_{k}^{T} A^{T} D_{k}^{-1} C_{k} A p_{k} \leq \alpha_{k}\left(\psi_{k}\left(p_{k}\right)+\frac{1}{2} p_{k}^{T} A^{T} D_{k}^{-1} C_{k} A p_{k}\right) .
$$

Clearly (AS.3) is satisfied with $\alpha_{k} p_{k}$ for sufficiently large $k$. From $0<\beta_{c s}<1$ and $\left\|g_{k}^{*}\right\| \leq \Delta_{k}$, for $k$ sufficiently large,

$$
\psi_{k}\left(\alpha_{k} p_{k}\right)+\frac{\alpha_{k}^{2}}{2} p_{k}^{T} A^{T} D_{k}^{-1} C_{k} A p_{k}<\beta_{c s}\left(\psi_{k}\left(g_{k}^{*}\right)+\frac{1}{2} g_{k}^{* T} A^{T} D_{k}^{-1} C_{k} A g_{k}^{*}\right),
$$

i.e., $\alpha_{k} p_{k}$ satisfies (AS.1). Since the first order necessary conditions are satisfied at $\left(x_{*} ; \lambda_{*}\right)$ with strict complementarity, $g_{k}=\tilde{g}_{k}$ for sufficiently large $k$. Thus $\alpha_{k} p_{k}$ satisfies (AS.2) asymptotically as well.

Following Theorem 4.4, $\alpha_{k} p_{k}$ yields a successful step for sufficiently large $k$. Therefore $x_{k+1}=x_{k}+\alpha_{k} p_{k}$ asymptotically. Using Theorem 4.4 again, $\left\{\left(x_{k} ; \lambda_{k}^{p}\right)\right\}$ converges quadratically to $\left(x_{*} ; \lambda_{*}\right)$.

It is straightforward to verify that the convergence analysis and results hold if, in the computation of the projected gradient $g_{k}, D_{k}^{\frac{1}{2}}$ is replaced by $D_{k}^{l}$ where $l \geq \frac{1}{2}$. Similar remarks can be made for $\tilde{g}_{k}$.

5. Conclusion. In this paper, global and local convergence properties of a trust region and affine scaling interior point method (TRAM) are established for minimizing a general nonlinear (nonconvex) function with linear inequality constraints.

Under compactness and full row rank assumption (AS.0), it is established that, if a computed step leads to a sufficient decrease with respect to the projected gradient $g_{k}$ using affine scaling $D_{k}$, every limit point satisfies the complementarity conditions. If (AS.2) is satisfied asymptotically, then there exists a limit $x_{*}$ with $\nabla f_{*}^{T} \tilde{g}_{*}=0$. If, in addition, strict complementarity holds at $x_{*}$. Then $\nabla f_{*}^{T} \tilde{g}_{*}=0$ at every limit point of $\left\{x_{k}\right\}$. Thus the Kuhn-Tucker conditions are satisfied at any limit $x_{*}$ satisfying strict complementarity. Moreover, if (AS.3) is satisfied asymptotically, then there exists a limit point at which both the first and second order necessary conditions are satisfied. Finally, if the second order sufficiency conditions and strict complementarity are satisfied at a limit point, then $\left\{\left(x_{k} ; \lambda_{k}^{p}\right)\right\}$ converges quadratically.

In the proposed method, a 2-norm trust region subproblem can be approximately solved. This trust region subproblem uses an affine scaling so that a damped step 
of the subproblem solution generates sufficient decrease of the objective function. Moreover, asymptotically, the solution of the trust region subproblem corresponds to an approximate Newton step for complementarity conditions. Preliminary computational results in [8] suggest that the proposed method is capable of solving many large linear-inequality-constrained nonlinear minimization problems in small numbers of iterations. 


\section{REFERENCES}

[1] N. K. Adler, M. Resende, AND G. Veiga, An implementation of karmarkar's algorithm for linear programming, Mathematical Programming, 44 (1989).

[2] M. A. BRANCh, T. F. Coleman, AND Y. LI, A subspace, interior and conjugate gradient method for large-scale bound-constrained minimization, Tech. Report TR95-1525, Computer Science Department, Cornell University, 1995.

[3] R. H. Byrd, J. C. Gilbert, AND J. Nocedal, A trust region method based on interior point techniques for nonlinear programming, Tech. Report OTC96/02, Optimization Technology Center, Northwestern University, 1996.

[4] Y.-C. Chen, D. J. Houck, J.-M. Liu, M. Meketon, R. J. V. L. Slutsman, and P. Wang, The ARET KORBX System, AT\&T Tecnnical Journal, (1989).

[5] T. F. Coleman AND Y. LI, A global and quadratically-convergent method for linear $l_{\infty}$ problems, SIAM Journal on Scientific and Statistical Computing, 29 (1992), pp. 1166-1186.

[6] — A globally and quadratically convergent affine scaling method for linear $l_{1}$ problems, Mathematical Programming, 56, Series A (1992), pp. 189-222.

[7] — An interior, trust region approach for nonlinear minimization subject to bounds, SIAM Journal on Optimization, 6 (1996).

[8] — Combining trust region and affine scaling for linearly constrained nonconvex minimization, Tech. Report TR97-1641, Computer Science Department, Cornell University, 1997. Submitted to the Proceeding of the International Nonlinear Programming Conference held in Beijing, September 2-5, 1996.

[9] T. F. Coleman and J. LIU, An interior Newton method for quadratic programming, Tech. Report TR93-1388, Computer Science Department, Cornell University, 1993.

[10] J. E. Dennis, M. H. JR., AND L. N. VICENTE, Trust-region interior-point sqp algorithms for a class of nonlinear programming problems, Tech. Report TR94-45, Department of Computational and Applied Mathematics, Rice University, 1994.

[11] J. E. Dennis AND J. J. MoRÉ, Quasi-Newton methods, motivation and theory, SIAM Review, 19 (1977), pp. 46-89.

[12] I. DIKIN, Iterative solution of problems of linear and quadratic programming, Doklady Akademiia Nauk SSSR, 174 (1967), pp. 747-748.

[13] A. S. El-Bakry, R. A. TAPia, T. Tsuchiya, And T. Zhang, On the formulation and theory of the Newton interior-point method for nonlinear programming, (1992).

[14] R. Fletcher, Practical Methods of Optimization: Volume 2, Constrained Optimization, John Wiley and Sons, 1981.

[15] A. Forsgren AND P. E. GILL, Primal-dual interior methods for nonconvex nonlinear programming, Tech. Report NA 96-3, Department of Mathematics, University of California, San Diego, 1996.

[16] C. C. GonZaga, Path following methods for linear programming, SIAM Review, 34 (1992), pp. $167-227$.

[17] R. KRANICH, Interior point methods for mathematical programming: A bibliograph, tech. report, Available through NETLIB: send e-mail to netlib@research.att.com containing the message "send intbib.bib from bib.

[18] Y. LI, A Newton acceleration of the Weiszfeld algorithm for minimizing the sum of Euclidean distances, Computational Optimization and Applications, to appear.

[19] Y. LI, A globally convergent method for $l_{p}$ problems, SIAM Journal on Optimization, (1993), pp. 609-629.

[20] Y. LI, A trust region and affine scaling method for nonlinearly constrained minimization, Tech. Report TR 94-1463, Computer Science Department, Cornell University, 1994, (Submitted to SIAM J. Optimization).

[21] R. Monteriro, T. TsuchiYa, And Y. WANG, A simplified global convergence proof of the affine scaling algorithm, Annals of Operation Research, (1993).

[22] J. J. MoRÉ, Recent developments in algorithms and software for trust region methods, in Mathematical Programming: The State of the Art, M. G. A. Bachem and B. Korte, eds., Springer Verlag, Berlin, 1983.

[23] J. J. MorÉ AND D. Sorensen, Computing a trust region step, SIAM Journal on Scientific and Statistical Computing, 4 (1983), pp. 553-572.

[24] M. ToDD, Potential-reduction methods in mathematical programming, Tech. Report TR1112, School of Operations Research and Industrial Engineering, Cornell University, 1995. 
[25] T. Tsuchiya AND R. Monteriro, Superlinear convergence of the affine scaling algorithm, Mathematical Programming, (1996).

[26] R. J. Vanderbei, M. S. Meketon, and B. A. Freedman, A modification of Karmarkar's linear programming algorithm, Algorithmica, 1 (1986), pp. 395-407. 